\title{
Chemical derivatization of phomosine A, a highly antifungal secondary metabolite from Phomopsis sp.
}

\author{
Karsten Krohn, ${ }^{\text {a }}$ Hidayat Hussain, ${ }^{a}$ Hans Egold, ${ }^{a}$ Barbara Schulz, ${ }^{\text {b }}$ and Ivan Green ${ }^{c_{*}}$ \\ ${ }^{a}$ Department of Chemistry, University of Paderborn, Warburger Straße 100, \\ 33098 Paderborn, Germany \\ ${ }^{b}$ Institute of Microbiology, University of Braunschweig, Spielmannstraße 7, \\ 38106 Braunschweig, Germany \\ ${ }^{c}$ Department of Chemistry, University of the Western Cape, P/Bag x17, \\ Bellville, 7530, South Africa \\ E-mail: karsten.krohn@uni-paderborn.de, igreen@uwc.ac.za
}

\begin{abstract}
Phomosine $\mathrm{A}$ is an antifungal biaryl ether produced in large amounts by different strains of fungal Phomopsis endophytes. In this paper, the functional groups of the natural product are systematically transformed into derivatives to probe their effect on biological activity. Acetylation of the three phenolic hydroxyl groups of phomosine A (1) produced the acetates 2a-c and revealed their relative chemical reactivity to increase in the following order: 3 '-OH $<6-\mathrm{OH}<$ 4-OH. Allylation of 1 using silver (I) oxide as the mediator afforded the three $O$ - and C-4'-allyl ethers 3a-c in addition to the diallyl ether $\mathbf{4 a}$. Under basic conditions the tri- $O$-allyl derivate $\mathbf{4 b}$ was produced. The aldehyde group of $\mathbf{1}$ and $\mathbf{2 a}$ and $\mathbf{2 b}$ was transformed into the oximes or oxime ethers 5, 6a-d, 7, and 8a-c by reaction with hydroxylamine followed by subsequent alkylation or by reaction with methoxyhydroxylamines. The hydrazones $9 \mathbf{a}$ and $\mathbf{9 b}$ and imines $9 \mathbf{c}$ and $9 \mathbf{d}$ were prepared by similar reactions. Borohydride reduction of $\mathbf{1}$ and the diacetate $\mathbf{2 b}$ produced the benzyl alcohols 10a and 10b respectively. The latter compound viz., 10b was converted into the benzyl ethers $\mathbf{1 1 a}$ and $\mathbf{1 1 b}$. Interestingly, in the triacetate $\mathbf{2 a}$, the aldehyde group was reduced into a methyl group in the products 12a-d. All the derived products were biologically active against test organisms for antibacterial, antifungal and antialgal activities.
\end{abstract}

Keywords: Antimicrobial agents, Semisynthesis, Phomosine A, Phomopsis species

\section{Introduction}

Phomopsis species are particularly productive in the synthesis of large arrays of structurally diverse secondary metabolites. ${ }^{1,2}$ We recently reported the isolation of phomosine A (1) (Scheme 
1), a highly antifungal biaryl ether aldehyde, from three different Phomopsis species. ${ }^{2-4}$ In this paper, we describe the synthesis of semi-synthetically prepared derivatives of phomosine A (1) to have a small assembly of derivatives to investigate the structure-activity relationship (SAR) of this potent antifungal drug. There are two major different functional elements contained in the structure of phomosine A (1), that allow for chemical modification. Firstly, it was also considered that the three phenolic $\mathrm{OH}$ groups would exhibit a relative different rate in chemical reactivity and secondly the aldehyde functional group allowed for a wide range of chemical manipulation. All of the semisynthetic products produced were evaluated against test organisms for antibacterial, antifungal and antialgal activities.

\section{Results and Discussion}

\section{Reactions at the phenolic hydroxyl groups}

To probe the biological influence of the three different phenolic hydroxyl groups in phomosine A (1), sequential blocking of these groups as an ester or ether would be desirable. Thus, the first experiments were aimed at chemical selectivity in both acylation and ether formation. Thus conversion of $\mathbf{1}$ to the triacetate $\mathbf{2 a}$, without reaction of the aldehyde group, was achieved by reaction of 1 with a large excess of acetic anhydride in pyridine. The conversion was complete within $2 \mathrm{~h}$ at $20{ }^{\circ} \mathrm{C}$ and $2 \mathbf{a}$ could be isolated in essentially quantitative yield (Scheme 1). To increase the selectivity, the reaction was next conducted in dilute dichloromethane solution in the presence of the sterically hindered and more selective lutidine as the base. In fact, after $18 \mathrm{~h}$ of stirring at $20^{\circ} \mathrm{C}$, a mixture of the less polar monoacetate $2 \mathrm{c}(11 \%)$ and the more polar diacetate $\mathbf{2 b}(79 \%)$ was isolated after chromatographic separation on silica gel.<smiles>COC(=O)c1c(C)c(Oc2cc(C)cc(O)c2C=O)c(O)c(C)c1O</smiles>

1<smiles>[R20]Oc1cc(C)cc(Oc2c([R20])c(C)c([R20])c(C(=O)OC)c2C)c1C</smiles>

2a: $\mathrm{R}^{1}=\mathrm{R}^{2}=\mathrm{R}^{3}=\mathrm{Ac}$

2b: $\mathrm{R}^{1}=\mathrm{H} ; \mathrm{R}^{2}=\mathrm{R}^{3}=\mathrm{Ac}$

2c: $\mathrm{R}^{1}=\mathrm{R}^{2}=\mathrm{H} ; \mathrm{R}^{3}=\mathrm{Ac}$

Scheme 1. Acetylation of phomosine A (1) to the phomosine A acetates 2a-c.

The following arguments and NMR experiments proved the regiochemistry of the acetates $\mathbf{2 b}$ and $\mathbf{2 c}$. In the diacetate $\mathbf{2 b}$, only one signal for a strongly chelated $\mathrm{OH}$ group at $\delta=12.0$ is 
visible. In the $\mathrm{HMBC}$ spectrum, this $\mathrm{OH}$ group correlates with the carbon atom appearing at $\delta=$ 163.5. This carbon further correlates in the HMBC spectrum with the formyl proton and the proton at $\delta=6.46$, vicinal to the free $\mathrm{OH}$ group, thus proving the position of the free hydroxyl group at C-3 $\square$. For the mono acetate 2c, signals for the two most shielded chelated hydroxyl groups at $\delta=11.69(6-\mathrm{OH})$ and $11.91\left(3^{\prime}-\mathrm{OH}\right)$ appear with similar chemical shifts to those in the trisphenol 1 ( $\delta=11.87$ and 11.92 , respectively). ${ }^{3}$

Not unexpectedly, the sequence of reactivity of the three phenolic groups was thus established. The hydroxyl group at C-3', being strongly chelated with the neighboring aldehyde function, was the least reactive, followed by the 6-OH, sterically shielded by two neighboring groups, but still being chelated to the ortho ester group at C-1. In spite of steric hindrance, the most reactive phenolic hydroxyl group was the non-chelated one at $\mathrm{C}-4$.

In the next series of reactions, we investigated protection of the phenolic hydroxyl groups with the allyl moiety since this in turn could be removed by palladium-catalyzed reactions if required. Two different methods were employed to achieve this. Firstly, reaction with allyl bromide in diethyl ether in the presence of active silver (I) oxide and secondly by alkylation with allyl bromide in DMF in the presence of the base potassium carbonate.

Under the silver oxide mediated reaction conditions, four products viz., 3a-c and $\mathbf{4 a}$ were isolated in which 3a-c had undergone both $\mathrm{C}$-allylation at $\mathrm{C}-4^{\prime}$, the sterically least hindered free position and the desired $O$-allylations to different degrees (Scheme 2).

1

a) $\mathrm{Ag}_{2} \mathrm{O}$

b) $\mathrm{K}_{2} \mathrm{CO}_{3}$<smiles>[R2]Oc1c(C)c([R20])c(Oc2cc(C)c(CC=C)c([R2])c2C=O)c(C)c1C(=O)OC</smiles>

3a: $\mathrm{R}^{1}=\mathrm{R}^{2}=\mathrm{H} ; \mathrm{R}^{3}=$ allyl

3b: $\mathrm{R}^{1}=\mathrm{H} ; \mathrm{R}^{2}=\mathrm{R}^{3}=$ allyl

3c: $\mathrm{R}^{1}=\mathrm{R}^{2}=\mathrm{R}^{3}=$ allyl<smiles>[R2]OC(=O)c1c(O[R])cc(C)cc1Oc1c(C)c(C(=O)O[R2])c([R2])c(C)c1C=O</smiles>

4a: $\mathrm{R}^{1}=\mathrm{H} ; \mathrm{R}^{2}=\mathrm{R}^{3}=$ allyl

4b: $\mathrm{R}^{1}=\mathrm{R}^{2}=\mathrm{R}^{3}=$ allyl

Scheme 2. Allylation of phomosine A (1) to the phomosine A allyl ethers $\mathbf{3 a - 4 b .}$

Evidently, the allylic cation generated by the action of silver oxide on allyl bromide was attacked by the aromatic nucleus in the meta position to the deactivating aldehyde group in agreement with the general rules for Friedel-Crafts alkylations. It is noteworthy that the least reactive chelated phenolic $\mathrm{OH}$ group at $\mathrm{C}-3^{\prime}$ followed by the second less reactive $\mathrm{OH}$ group at $\mathrm{C}$ 6 remained intact in the products $\mathbf{3 a}, \mathbf{3 b}$, and $\mathbf{4 a}$, respectively, supporting the earlier finding. On 
the other hand, under base-catalyzed $\left(\mathrm{K}_{2} \mathrm{CO}_{3}\right)$ conditions, no $C$-allylation occurred with only the three phenolic groups being allylated to yield $\mathbf{4 b}$.

\section{Reactions at the aldehyde functional group}

The aldehyde functional group in phomosine $\mathrm{A}$ is ideally suited for a great variety of modifications. In the initial experiments, the investigated reactions did not alter the oxidation state of the aldehyde. We were keen, firstly, to convert the aldehyde $\mathbf{1}$ into a number of oximes and oxime ethers. To this end phomosine A (1) was treated with hydroxylamine hydrochloride in ethanol $^{5-7}$ to afford the oxime $\mathbf{5}$ in $85 \%$ yield (Scheme 3 ).<smiles>CC(=O)c1c(C)c(Oc2cc(C)cc(O)c2C=NO)c(O)c(C)c1O</smiles><smiles>[R2]N=Cc1c(O[R])cc(C)cc1Oc1c(C)c(C(=O)O[R])c(O[14C]([R10])C)c(C)c1OC(C)=O</smiles>

6b: $\mathrm{R}=\mathrm{CH}_{3}$

6c: $\mathrm{R}=\mathrm{CH}_{2} \mathrm{CH}_{3}$

6d: $\mathrm{R}=\mathrm{Bn}$<smiles>COC(=O)c1c(C)c(Oc2cc(C)cc(O)c2C=NO)c(C)c(OC(C)=O)c1C(C)=O</smiles>

6a<smiles>CC(=O)Oc1c(C)c(Oc2cc(C)c(Cc3ccccc3)c(OCc3ccccc3)c2C=NO)c(C)c(C(C)=O)c1C</smiles><smiles>COC(=O)c1c(C)c(Oc2cc(C)cc(O)c2C=NO)c(O)c(C)c1O</smiles>

$8 \mathbf{a}$<smiles>[R]Oc1cc(C)cc(Oc2c(C)c(C(=O)OC)c(OC(C)=O)c(C)c2OC(C)=O)c1C=NOC</smiles>

8b: $\mathrm{R}=\mathrm{H}$

8c: $R=A c$

Scheme 3. Conversion of phomosine A (1) into its oxime 5 and $\mathbf{6 a}$ and oxime ethers $\mathbf{6 b - d , 7}$, and 8a-c. 
Since the diacetate $\mathbf{2 b}$ was available by our earlier selective acetylation, we also prepared the oxime diacetate $\mathbf{6 a}$ under the same conditions without saponification of the two acetate groups under the mildly acidic conditions employed. Interestingly, the same product viz., 6a was reproducibly obtained by reaction of the triacetate $\mathbf{2 a}$ with hydroxylamine hydrochloride under the same conditions. Evidently, the vicinal phenolic acetic ester group was cleaved by neighboring group participation of the formed oxime.

Two different methods are in principle available for the preparation of the oxime ethers viz., a) alkylation of the oxime hydroxyl group and b) reaction of the aldehyde with hydroxylamine ethers. Both methods proved to be successful in our hands. Methylation of the oxime diacetate 6a with methyl iodide and silver (I) oxide as the mediator gave the oxime methyl ether $\mathbf{6 b}$ in which the free hydroxyl group at C-3' was also methylated (Scheme 3). Similar reactions were also realized with ethyl iodide and benzyl bromide which afforded the ethyl and benzyl ethers $\mathbf{6 c}$ and $\mathbf{6 d}$ respectively. In the latter reaction with benzyl bromide, the C-4' benzylated product 7 was also formed similarly found for the allylation reactions (Scheme 3). An alternative route to the oxime methyl ether 8a was realized in the conversion of phomosine A (1) by reaction with $O$-methylhydroxylamine in ethanol. Similar conversions were effected by treatment of the diacetate $\mathbf{2 b}$ and triacetate $\mathbf{2 a}$ under similar conditions to afford the corresponding hydroxylamine methyl ethers $\mathbf{8 b}$ and $\mathbf{8 c}$ respectively (Scheme 3).

The last transformations with the unchanged oxidation state of the aldehyde involved preparation of hydrazones and imines. Reaction of aldehyde 1 with methyl hydrazine carboxylate in ethanol afforded the corresponding hydrazone carboxylate 9a. A similar conversion was realized by treatment of $\mathbf{1}$ with 1,1-dimethyl hydrazine to yield the dimethyl hydrazone $\mathbf{9 b}$.

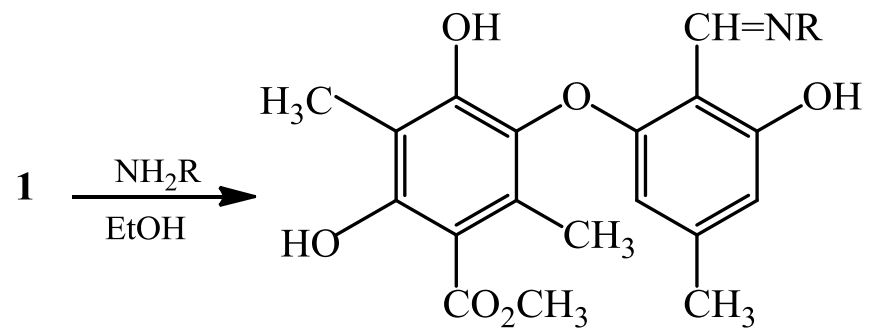

$$
\begin{aligned}
& \text { 9a: } \mathrm{R}=\mathrm{NHCO}_{2} \mathrm{CH}_{3} \\
& \text { 9b: } \mathrm{R}=\mathrm{N}\left(\mathrm{CH}_{3}\right)_{2} \\
& \text { 9c: } \mathrm{R}=\mathrm{CH}_{2} \mathrm{CH}_{2} \mathrm{CH}_{3} \\
& \text { 9d: } \mathrm{R}=\mathrm{CH}_{2} \mathrm{Ph}
\end{aligned}
$$

Scheme 4. Conversion of $\mathbf{1}$ into the hydrazones $\mathbf{9 a , b}$ and imines $\mathbf{9 c , d}$.

The related imines 9c and 9d were obtained by reaction of $\mathbf{1}$ with either 1-propylamine or benzylamine. Since all these reactions were thermodynamically controlled, the more stable trans hydrazones and imines were expected to be formed (Scheme 4).

Finally, in the last experiments, the oxidation state of the aldehyde group was changed by reduction. As expected, reduction of the parent aldehyde phomosine A (1) was straightforward to afford the benzyl alcohol 10a in quantitative yield. Similarly, the reduction of the 4,6-diacetate 2b furnished the bis-acetylated benzyl alcohol 10b. Next, the selective protection of the two hydroxyl groups in 10b viz., benzylic and phenolic was exploited. Thus treatment of $\mathbf{1 0 b}$ with 
benzyl bromide in the presence of silver (I) oxide produced the 3'-monobenzylated benzyl alcohol 11a in $72 \%$ yield. The selective benzylation of the phenolic group was unambiguously proven by NMR analysis and comparison with authentic isomeric benzyl ethers. ${ }^{2}$ The chemical shift of the carbon atom in the free benzyloxy showed the typical value of $54.7 \mathrm{ppm}$ in the ${ }^{13} \mathrm{C}$ NMR spectrum, whereas the isomeric benzyl ether had lower field values around 63-64 ppm. ${ }^{2}$ The final conversion of this molecule involved Zemplen deacetylation of 11a with $\mathrm{NaOMe}$ in methanol which produced the trihydroxy analogue 11b (Scheme 5).<smiles>[2H]c1c(C)c(Oc2ccccc2)c(Oc2cc(C)cc(O)c2CO)c(C)c1C(=O)O[18F]</smiles>

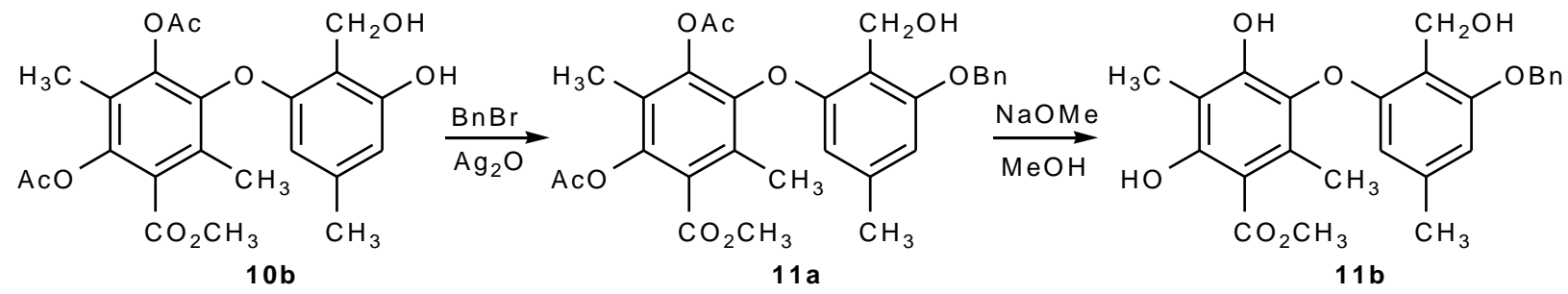<smiles>CC(=O)Oc1cc(C)cc(Oc2c(C)c(OC(C)=O)c(OC(C)=O)c(C(C)=O)c2C)c1C=O</smiles>

2a<smiles>CC(=O)c1c(C)c(Oc2cc(C)cc(O)c2C)c(O)c(C)c1O</smiles>

$12 \mathrm{a}-\mathrm{d}$

\begin{tabular}{llll} 
& $R^{1}$ & $R^{2}$ & $R^{3}$ \\
\hline 12a: & $A c$ & $A c$ & $A c$ \\
12b: & $A c$ & $H$ & $A c$ \\
12c: & $H$ & $A c$ & $A c$ \\
12d: & $H$ & $H$ & $A c$
\end{tabular}

Scheme 5. Reduction of phomosine A (1) and its acetates $\mathbf{2 a}$ and $\mathbf{2 b}$ to the corresponding hydroxymethyl or methyl compounds 10-12.

Quite surprising results were obtained in the borohydride reduction of the triacetate $\mathbf{2 a}$. In this instance four different products 12a-d were isolated from the reaction mixture. In contrast to the reduction of the aldehyde group of phomosine A (1) and its 4,6-diacetate $\mathbf{2} \mathbf{b}$, the aldehyde group of the triacetylated phomosine A (2a) was fully reduced to a methyl group. The mixture of phenols 12b-d presumably resulted from partial saponification of the initially formed triacetate 12a (Scheme 5). Assignments for 12b, 12c and 12d was based on the $\delta$ values for the 2-OH and 3'-OH as compared to the literature. ${ }^{3}$ 
Table 1. Biological activities of the derivatives of phomosine A at a concentration of $50 \mu \mathrm{g}$ against microbial test organisms in an agar diffusion assay

\begin{tabular}{|c|c|c|c|c|}
\hline $\begin{array}{l}\text { Substanc } \\
\text { e }\end{array}$ & $\begin{array}{c}\text { Bacillus } \\
\text { megaterium }\end{array}$ & E. coli K 12 & $\begin{array}{c}\text { Microbotryum } \\
\text { violaceum }\end{array}$ & $\begin{array}{c}\text { Chlorella } \\
\text { fusca }\end{array}$ \\
\hline $\mathbf{1}^{4}$ & 11 & - & PI 7 & 5 \\
\hline $2 \mathbf{a}$ & PI 6 & 6 & 7 & 6 \\
\hline $2 b$ & PI 5.5 & PI 6 & 5.5 & 5.5 \\
\hline $2 c$ & PI 6.5 & PI 5 & 6 & 5 \\
\hline $3 \mathbf{b}$ & PI 5.5 & 6.5 & 5.5 & $5.5+$ PI 6 \\
\hline $3 c$ & PI 6 & PI 5.5 & 6 & 5.5 \\
\hline $\mathbf{4 a}$ & 8 & PI 5 & 5.5 & 5.5 \\
\hline $4 b$ & PI 6.5 & PI 5 & 7 & 6 \\
\hline 5 & PI 6 & PI 5 & 6 & 6 \\
\hline $6 \mathbf{a}$ & PI 6 & 5 & $6+$ PI 12 & 5.5 \\
\hline $\mathbf{6 b}$ & PI 6 & PI 5 & 6 & 6 \\
\hline $6 c$ & PI 6 & 5.5 & 6 & 6 \\
\hline $6 d$ & PI 5.5 & 6 & 6 & 5.5 \\
\hline 7 & PI 6 & 5 & 6 & 5 \\
\hline $8 \mathbf{a}$ & PI 5.5 & 5 & 6.5 & 5 \\
\hline $8 b$ & PI 6 & PI 5.5 & 5.5 & 6 \\
\hline $8 c$ & PI 7 & PI 5.5 & 6.5 & 6 \\
\hline $9 \mathbf{a}$ & PI 6.5 & 6 & 6.5 & 5 \\
\hline $9 b$ & PI 6 & PI 6 & 6 & 5.5 \\
\hline $9 c$ & PI 4.5 & PI 6 & 6 & 5 \\
\hline 9d & PI 5.5 & PI 6 & 5.5 & 5 \\
\hline $10 b$ & PI 6 & PI 5.5 & 5.5 & 6 \\
\hline $11 a$ & PI 6 & PI 5 & 6 & 5.5 \\
\hline $11 b$ & PI 5 & PI 5 & 6 & 5.5 \\
\hline $12 \mathbf{a}$ & PI 6 & PI 6 & 6.5 & 5.5 \\
\hline $12 \mathrm{c}$ & PI 6 & 5 & 5.5 & 0 \\
\hline
\end{tabular}


Table 1 (continued)

\begin{tabular}{lcccc}
\hline Substance & $\begin{array}{c}\text { Bacillus } \\
\text { megaterium }\end{array}$ & E. coli K 12 & $\begin{array}{c}\text { Microbotryum } \\
\text { violaceum }\end{array}$ & $\begin{array}{c}\text { Chlorella } \\
\text { fusca }\end{array}$ \\
\hline $\mathbf{1}^{4}$ & 11 & - & PI 7 & 5 \\
\hline $\mathbf{1 2 d}$ & PI 6 & 5 & 6 & 5.5 \\
acetone & 0 & 0 & 0 & 0 \\
penicillin & 18 & 14 & 0 & 0 \\
tetracycline & 18 & 18 & 0 & PI 10 \\
nystatin & 0 & 0 & 20 & 0 \\
actidione & 0 & 0 & 50 & 35 \\
\hline
\end{tabular}

${ }^{a} 50 \mu \mathrm{g}$ each of compound (2a-12d) and of the four control compounds were tested in an agar diffusion assay for inhibitions of Bacillus megaterium, Escherichia coli, Chlorella fusca and Microbotryum violaceum. The radius of zone of inhibition was measured in $\mathrm{mm}$. PI = partial inhibition, i.e. there was some growth within the zone of inhibition.

\section{Biological Activity}

The substances were tested in an agar diffusion assay for antimicrobial activity. As displayed in Table 1, all of the tested substances were moderately biologically active against all of the test organisms viz., antifungal against Microbotryum violaceum, antibacterial against the gram negative bacterium Escherichia coli as well as the gram positive bacterium Bacillus megaterium, and antialgal against Chlorella fusca. However, none of the derivatives was as active as phomosine A (1). ${ }^{2-4}$

\section{Experimental Section}

General Procedures. Melting points were determined with a Gallenkamp melting point apparatus. The IR spectra were recorded with a Nicolet-510P spectrometer. NMR spectra were recorded with a Bruker Avance-500 NMR spectrometer with TMS as internal standard. Assignment of NMR signals are based on the 2D spectra. EI mass spectra were obtained with a Thermo Finnigan MAT 8200 mass spectrometer.

General procedure for phomosine A derivatives:

3',4,6-Triacetylphomosine A (2a): A solution of phomosine A (1) (100 mg; $0.29 \mathrm{mmol})$ in acetic anhydride $(4 \mathrm{ml})$ and pyridine $(1 \mathrm{ml})$ was stirred for $2 \mathrm{~h}$ at $25^{\circ} \mathrm{C}$. Iced water $(50 \mathrm{ml})$ was then added and the solution was stirred for $30 \mathrm{~min}$. A white precipitate was formed which was filtered off and washed with water to afford the triacetate as white crystals (136 mg, 100\%), m.p. 
166-167 ${ }^{\circ} \mathrm{C}$. IR $\nu_{\max }\left(\mathrm{CH}_{2} \mathrm{Cl}_{2}\right): 2860,1630,1533,1297 \mathrm{~cm}^{-1} .{ }^{1} \mathrm{H}$ NMR $\left(500 \mathrm{MHz}, \mathrm{CDCl}_{3}\right): \delta=$ $1.98,2.04,2.25,2.29,2.31$ and 2.38 (s, each $3 \mathrm{H}, 4-\mathrm{CH}_{3} \mathrm{CO}, 6-\mathrm{CH}_{3} \mathrm{CO}, 3{ }^{\prime}-\mathrm{CH}_{3} \mathrm{CO}, 2-\mathrm{CH}_{3}, 5-$ $\mathrm{CH}_{3}$ ), 3.92 (s, $3 \mathrm{H}, 1-\mathrm{CO}_{2} \mathrm{CH}_{3}$ ), 6.30 (s, $\left.1 \mathrm{H}, 6^{\prime}-\mathrm{H}\right), 6.60$ (s, $\left.1 \mathrm{H}, 4^{\prime}-\mathrm{H}\right), 10.51$ (s, $\left.1 \mathrm{H}, 2^{\prime}-\mathrm{CHO}\right)$. ${ }^{13} \mathrm{C}-\mathrm{NMR}\left(125 \mathrm{MHz}, \mathrm{CDCl}_{3}\right): \delta=10.5\left(5-\mathrm{CH}_{3}\right), 13.7\left(2-\mathrm{CH}_{3}\right), 21.0\left(5^{\prime}-\mathrm{CH}_{3}\right), 20.4,19.9,22.1$ (4$\mathrm{CH}_{3} \mathrm{CO}, 6-\mathrm{CH}_{3} \mathrm{CO}, 3{ }^{\prime}-\mathrm{CH}_{3} \mathrm{CO}$,), $52.5\left(1-\mathrm{CO}_{2} \mathrm{CH}_{3}\right), 112.8,115.1,118.6(\mathrm{CH}), 125.2,125.9$, $129.4(\mathrm{CH}), 142.4,143.8,144.8,148.0,150.5160 .7,166.0,167.4,168.3,169.5$, and 186.9. EIMS $(\mathrm{m} / \mathrm{z}) \%=472.1(20), 430.1$ (58), 388.1 (100), 346.1 (76), 314.1 (83), $286.1(17), 257.1$ (8), 193.1 (13), 151.0 (58), 83 (28). HREIMS: $m / z 472.1368$ (calcd. 472.1370 for $\mathrm{C}_{24} \mathrm{H}_{24} \mathrm{O}_{10}$ )

4,6-Diacetyl and 4-acetyl phomosine A (2b and 2c): A solution of phomosine A (1) (274 mg, $0.79 \mathrm{mmol})$ in dichloromethane $(13 \mathrm{ml})$ treated with acetic anhydride $(2.4 \mathrm{~g} ; 2.22 \mathrm{ml} ; 2.35$ $\mathrm{mmol})$ and 2,6-lutidine $(0.07 \mathrm{~g} ; 0.07 \mathrm{ml} ; 0.07 \mathrm{mmol})$ was stirred under argon until the starting material was consumed $18 \mathrm{~h}$ (TLC monitoring). The solution was diluted with additional $\mathrm{CH}_{2} \mathrm{Cl}_{2}$ $(50 \mathrm{ml})$ and then intensely washed with water $(3 \times 25 \mathrm{ml})$. The organic phase was dried $\left(\mathrm{Na}_{2} \mathrm{SO}_{4}\right)$, the solvent removed under reduced pressure and the residue obtained was chromatographed and eluted with EtOAc- $n$-hexane (7:3) to yield as the first fraction the phomosine A 4-monoacetate (2c) (34 mg; 11\%) and phomosine A 4,6-diacetate (2b) (270 mg; $79 \%)$.

4,6-Diacetyl phomosine A (2b): White crystals m.p. $155-156^{\circ} \mathrm{C}$ (from EtOAc-hexane). IR $v_{\max }$ $\left(\mathrm{CH}_{2} \mathrm{Cl}_{2}\right): 3330,2870,1640,1530,1290 \mathrm{~cm}^{-1} .{ }^{1} \mathrm{H} \mathrm{NMR}\left(500 \mathrm{MHz}, \mathrm{CDCl}_{3}\right): \delta=2.04,2.13,2.27$ (×2) and 2.36 (each $3 \mathrm{H}$, s, Aryl- $\mathrm{CH}_{3}$ and $\left.\mathrm{CH}_{3} \mathrm{CO}\right), 3.97\left(\mathrm{~s}, 3 \mathrm{H}, \mathrm{OCH}_{3}\right), 5.81\left(\mathrm{~s}, 1 \mathrm{H}, 6^{\prime}-\mathrm{H}\right), 6.46$ (s, $\left.1 \mathrm{H}, 4^{\prime}-\mathrm{H}\right), 10.45(\mathrm{~s}, 1 \mathrm{H}, \mathrm{CHO})$ and $12.0\left(\mathrm{~s}, 1 \mathrm{H}, 3^{\prime}-\mathrm{OH}\right) .{ }^{13} \mathrm{C} \mathrm{NMR}\left(125 \mathrm{MHz}, \mathrm{CDCl}_{3}\right): \delta=$ 10.5, 13.6, 20.1, 20.4 and $22.7\left(\mathrm{CH}_{3}\right), 52.5\left(\mathrm{OCH}_{3}\right), 104.9\left(\mathrm{C}-4^{\prime}\right), 108.7,112.0(\mathrm{C}-6), 125.0$, $125.9,129.4,142.0,143.9,144.8,151.0,159.5,163.5,166.0,167.3,168.3$ and 192.9.. EIMS $\mathrm{m} / z$ $(\%)=430.1$ (23), 388.1 (26), 346.1 (66), 286.1 (18), 258.0 (5), 151.0 (45). HREIMS: $\mathrm{m} / z$ 430.1254 (calcd. 430.1264 for $\mathrm{C}_{22} \mathrm{H}_{22} \mathrm{O}_{9}$ ).

4-Acetyl phomosine A (2c): White crystals, m.p. $179^{\circ} \mathrm{C}$ (from EtOAc-hexane). IR $v_{\max }$ $\left(\mathrm{CH}_{2} \mathrm{Cl}_{2}\right): 3420,2860,1630,1533,1297 \mathrm{~cm}^{-1} .{ }^{1} \mathrm{H}$ NMR $\left(500 \mathrm{MHz}, \mathrm{CDCl}_{3}\right): \delta=2.09$, 2.19, and 2.37(x2) (s, each $3 \mathrm{H}$ Aryl- $\mathrm{CH}_{3}$ and $\left.\mathrm{CH}_{3} \mathrm{CO}\right), 3.99$ (s, $3 \mathrm{H}, \mathrm{OCH}_{3}$, 5.69 (s, $\left.1 \mathrm{H}, 6^{\prime}-\mathrm{H}\right), 6.41$ (s, 1 $\left.\mathrm{H}, 4^{\prime}-\mathrm{H}\right), 10.45$ (s, $\left.1 \mathrm{H}, \mathrm{CHO}\right), 11.69$ (s, $\left.1 \mathrm{H}, 6-\mathrm{OH}\right), 11.91$ (s, $\left.1 \mathrm{H}, 3^{\prime}-\mathrm{OH}\right) .{ }^{13} \mathrm{C}$ NMR (125 $\left.\mathrm{MHz}, \mathrm{CDCl}_{3}\right): \delta=9.5,15.2,20.1,22.7\left(\mathrm{CH}_{3}\right.$ and $\left.\mathrm{CH}_{3} \mathrm{CO}\right), 52.5\left(\mathrm{OCH}_{3}\right), 104.7\left(\mathrm{C}^{-} 6^{\prime}\right), 108.7$, 110.4, 111.6 (C-4'), 119.3, 131.7, 136.5, 146.9, 150.7, 159.7, 160.4, 163.5, 167.4, 171.9 and 193.0.. EIMS $m / z=387.9$ (66), 346.0 (43), 313.9 (100), 285.9 (26), 258.0 (7), 150.9 (46). HREIMS: $m / z 388.1157$ (calcd. 388.1158 for $\mathrm{C}_{20} \mathrm{H}_{20} \mathrm{O}_{8}$ ).

Allylation of phomosine A (1): A solution of phomosine A (1) (100 mg; $0.29 \mathrm{mmol})$ in diethyl ether $(10 \mathrm{ml})$ was treated with allyl bromide $(350 \mathrm{mg} ; 2.9 \mathrm{mmol} ; 0.251 \mathrm{ml})$ and argentous oxide (673 mg; $2.9 \mathrm{mmol}$ ) and stirred until the starting material was consumed (TLC monitoring). The suspension was filtered, the solvent removed under reduced pressure, and the residue obtained by evaporation of solvent was carefully chromatographed and eluted with $n$-hexane-EtOAc (7:1) to afford the following order of products: 
4,4'-Diallylphomosine A (3a): $15 \mathrm{mg} ; 12 \%$. Colorless crystals m.p. $160-161^{\circ} \mathrm{C}$ (from hexane). IR $v_{\max }\left(\mathrm{CH}_{2} \mathrm{Cl}_{2}\right): 3310,2870,1650,1540,1290 \mathrm{~cm}^{-1} .{ }^{1} \mathrm{H}$ NMR $\left(500 \mathrm{MHz}, \mathrm{CDCl}_{3}\right): \delta=2.14$, 2.19 and 2.35 (each $3 \mathrm{H}, \mathrm{s}$, Aryl- $\mathrm{CH}_{3}$ ), 3.34 (dt, $J=6.0,1.5 \mathrm{~Hz}, 2 \mathrm{H}$, Aryl- $\mathrm{CH}_{2} \mathrm{CH}=\mathrm{CH}_{2}$ ), 3.97 $\left(\mathrm{s}, 3 \mathrm{H}, \mathrm{OCH}_{3}\right), 4.41\left(\mathrm{~d}, J=6.0 \mathrm{~Hz}, 2 \mathrm{H}, \mathrm{OCH}_{2} \mathrm{CH}=\mathrm{CH}_{2}\right), 4.97\left(\mathrm{~m}, 2 \mathrm{H}, \mathrm{CH}=\mathrm{CH}_{2}\right), 5.18(\mathrm{~m}, 2 \mathrm{H}$, $\left.\mathrm{CH}=\mathrm{CH}_{2}\right), 5.66\left(\mathrm{~s}, 1 \mathrm{H}, 6^{\prime}-\mathrm{H}\right), 5.88\left(\mathrm{~m}, 2 \mathrm{H}, 2 \times \mathrm{CH}=\mathrm{CH}_{2}\right), 10.53(\mathrm{~s}, 1 \mathrm{H}, \mathrm{CHO}), 11.66(\mathrm{~s}, 1 \mathrm{H}$, $\left.3^{\prime}-\mathrm{OH}\right), 12.23(\mathrm{~s}, 1 \mathrm{H}, 6-\mathrm{OH}) .{ }^{13} \mathrm{C}$ NMR (125 MHz, $\left.\mathrm{CDCl}_{3}\right): \delta=9.4,15.0$ and $20.8\left(\mathrm{CH}_{3}\right), 29.2$ $\left(\mathrm{CH}_{2} \mathrm{CH}=\mathrm{CH}_{2}\right), 52.2\left(\mathrm{OCH}_{3}\right), 74.6\left(\mathrm{OCH}_{2} \mathrm{CH}=\mathrm{CH}_{2}\right), 104.9(\mathrm{CH}), 108.4,108.8,114.7(\mathrm{CH})$, $118.3(\mathrm{CH}), 119.0,119.4,131.8,133.1(\mathrm{CH}), 135.4(\mathrm{CH}), 137.8,149.2,155.0,158.8,160.0$, 161.3, 172.1 and 193.0. EIMS $m / z=426.2$ (18), 394.2 (4), 346.2 (5), 314.1 (9), 297.1 (6), 237.1 6), 215.120 .0 ), 187.1 (15), 113.1 (73), 71.0 (100). (HRMS: Found: $426.16800 \mathrm{C}_{24} \mathrm{H}_{26} \mathrm{O}_{7}$ requires 426.16785).

4,4',6-Triallylphomosine A (3b): Colorless crystals (15 mg; 11\%) after column chromatography purification [ $n$-hexane-EtOAc (7:1)]. M.p. 85-86 ${ }^{\circ} \mathrm{C}$ (from hexane). IR $v_{\max }\left(\mathrm{CH}_{2} \mathrm{Cl}_{2}\right): 3210$, 2870, 1650, 1530, $1290 \mathrm{~cm}^{-1}$. ${ }^{1} \mathrm{H}$ NMR $\left(500 \mathrm{MHz}, \mathrm{CDCl}_{3}\right): \delta=2.16,2.18$ and 2.35 (each $3 \mathrm{H}$, s, Aryl- $\left.\mathrm{CH}_{3}\right), 3.38$ (dt, $J=5.5,1.5 \mathrm{~Hz}, 2 \mathrm{H}$, Aryl- $\left.\mathrm{CH}_{2} \mathrm{CH}=\mathrm{CH}_{2}\right), 3.96\left(\mathrm{~s}, 3 \mathrm{H}, \mathrm{OCH}_{3}\right), 4.45(\mathrm{dt}, J=$ $\left.5.5,1.5 \mathrm{~Hz}, 4 \mathrm{H}, 2 \times \mathrm{OCH}_{2} \mathrm{CH}=\mathrm{CH}_{2}\right), 4.90\left(\mathrm{dq}, J=17.0,1.5 \mathrm{~Hz}, 1 \mathrm{H}\right.$, trans $\left.-\mathrm{CH}=\mathrm{CH}_{2}\right), 5.01(\mathrm{dq}, J$ $=10.0,1.5 \mathrm{~Hz}, 1 \mathrm{H}$, cis $\left.-\mathrm{CH}_{2} \mathrm{CH}=\mathrm{CH}_{2}\right), 5.10\left(\mathrm{dq}, J=10.0,1.5 \mathrm{~Hz}, 1 \mathrm{H}\right.$, cis $\left.-\mathrm{CH}_{2} \mathrm{CH}=\mathrm{CH}_{2}\right), 5.19$ $\left(\mathrm{dq}, J 17.0,1.5 \mathrm{~Hz}, 1 \mathrm{H}\right.$, trans $\left.-\mathrm{CH}_{2} \mathrm{CH}=\mathrm{CH}_{2}\right), 5.29\left(\mathrm{dq}, J 10.0,1.5 \mathrm{~Hz}, 1 \mathrm{H}\right.$, cis $\left.-\mathrm{CH}_{2} \mathrm{CH}=\mathrm{CH}_{2}\right)$, $5.43\left(\mathrm{dq}, J 17.0,1.5 \mathrm{~Hz}, 1 \mathrm{H}\right.$, trans $\left.-\mathrm{CH}_{2} \mathrm{CH}=\mathrm{CH}_{2}\right), 5.88\left(\mathrm{~m}, 2 \mathrm{H}, 2 \times \mathrm{OCH}_{2} \mathrm{CH}=\mathrm{CH}_{2}\right), 6.02(\mathrm{~s}, 1$ $\left.\mathrm{H}, 6^{\prime}-\mathrm{H}\right), 6.14\left(\mathrm{~m}, 1 \mathrm{H}\right.$, Aryl- $\left.\mathrm{CH}_{2} \mathrm{CH}=\mathrm{CH}_{2}\right), 10.61(\mathrm{~s}, 1 \mathrm{H}, \mathrm{CHO})$ and $11.66\left(1 \mathrm{H}, \mathrm{s}, 3^{\prime}-\mathrm{OH}\right) .{ }^{13} \mathrm{C}$ NMR (125 MHz, $\left.\mathrm{CDCl}_{3}\right): \delta=9.3,15.1,20.6\left(\mathrm{CH}_{3}\right), 30.1\left(\right.$ Aryl $\left.-\mathrm{CH}_{2} \mathrm{CH}=\mathrm{CH}_{2}\right), 52.2\left(\mathrm{OCH}_{3}\right)$, 74.5 and $76.7\left(2 \times \mathrm{OCH}_{2} \mathrm{CH}=\mathrm{CH}_{2}\right), 108.3,111.1(\mathrm{CH}), 115.2(\mathrm{CH}), 116.5,117.9(\mathrm{CH}), 118.1$ $(\mathrm{CH}), 118.9,125.6,131.8,133.3(\mathrm{CH}), 133.4(\mathrm{CH}), 136.0(\mathrm{CH}), 138.2,147.0,154.8,158.5$, 159.6, 159.9, 172.2, 188.7. EIMS ( $m / z) \%=466.2$ (18), 426.1 (2), 393.1 (2), 337.1 (5), 279.1 (7), 231.1 (10), 179.0 (39), 117.0 (54), 91.0 (100). HREIMS: m/z 466.1992 (calcd. 466.1992for $\left.\mathrm{C}_{27} \mathrm{H}_{30} \mathrm{O}_{7}\right)$.

3',4,4',6-Tetraallylphomosine A (3c): Colorless oil (50 mg; 34\%) after column chromatography purification [ $n$-hexane-EtOAc (7:1)]. IR $v_{\max }\left(\mathrm{CH}_{2} \mathrm{Cl}_{2}\right): 2880,1650,1530,1290 \mathrm{~cm}^{-1}$. ${ }^{1} \mathrm{H}$ NMR $\left(500 \mathrm{MHz}, \mathrm{CDCl}_{3}\right): \delta=2.10,2.17$ and 2.20 (each $\left.3 \mathrm{H}, \mathrm{s}, \operatorname{Aryl}-\mathrm{CH}_{3}\right), 3.38(\mathrm{dt}, J=5.5,1.5 \mathrm{~Hz}, 2$ $\mathrm{H}$, Aryl- $\left.\mathrm{CH}_{2} \mathrm{CH}=\mathrm{CH}_{2}\right), 3.91\left(\mathrm{~s}, 3 \mathrm{H}, \mathrm{OCH}_{3}\right), 4.44\left(\mathrm{~m}, 6 \mathrm{H}, 3 \times \mathrm{OCH}_{2} \mathrm{CH}=\mathrm{CH}_{2}\right), 4.90(\mathrm{dq}, J=$ 17.0, $1.5 \mathrm{~Hz}, 1 \mathrm{H}$, trans $\left.-\mathrm{CH}_{2} \mathrm{CH}=\mathrm{CH}_{2}\right), 5.01\left(\mathrm{dq}, J=17.0,1.5 \mathrm{~Hz}, 1 \mathrm{H}\right.$, cis $\left.-\mathrm{CH}_{2} \mathrm{CH}=\mathrm{CH}_{2}\right), 5.10$ (dq, $J=17.0,1.5 \mathrm{~Hz}, 1 \mathrm{H}$, cis $\left.-\mathrm{CH}_{2} \mathrm{CH}=\mathrm{CH}_{2}\right), 5.18(\mathrm{dq}, J=17.0,1.5 \mathrm{~Hz}, 1 \mathrm{H}$, trans$\left.\mathrm{CH}_{2} \mathrm{CH}=\mathrm{CH}_{2}\right), 5.27\left(\mathrm{~m}, 2 \mathrm{H}, \mathrm{CH}_{2} \mathrm{CH}=\mathrm{CH}_{2}\right), 5.41\left(\mathrm{~m}, 2 \mathrm{H}, \mathrm{CH}_{2} \mathrm{CH}=\mathrm{CH}_{2}\right), 5.88(\mathrm{~m}, 2 \mathrm{H}$, $\left.2 \times \mathrm{CH}_{2} \mathrm{CH}=\mathrm{CH}_{2}\right), 6.05\left(\mathrm{~m}, 1 \mathrm{H}, \mathrm{CH}_{2} \mathrm{CH}=\mathrm{CH}_{2}\right), 6.07$ (s, $\left.1 \mathrm{H}, 6^{\prime}-\mathrm{H}\right), 6.13\left(\mathrm{~m}, 1 \mathrm{H}, \mathrm{CH}_{2} \mathrm{CH}^{\prime} \mathrm{CH}_{2}\right)$, 10.60 (s, $1 \mathrm{H}, \mathrm{CHO}) .{ }^{13} \mathrm{C}$ NMR (125 MHz, $\left.\mathrm{CDCl}_{3}\right): \delta=10.1,13.1,20.6\left(\mathrm{CH}_{3}\right), 30.3$ (Aryl$\left.\mathrm{CH}_{2} \mathrm{CH}=\mathrm{CH}_{2}\right), 52.4\left(\mathrm{OCH}_{3}\right), 74.4,75.3$ and $76.8\left(3 \times \mathrm{OCH}_{2} \mathrm{CH}=\mathrm{CH}_{2}\right), 111.2(\mathrm{CH}), 115.2(\mathrm{CH})$, 116.6, $117.4(\mathrm{CH}), 117.9(\mathrm{CH}), 118.0(\mathrm{CH}), 124.9,125.6,125.8,127.9,133.3(\mathrm{CH}), 133.4(\mathrm{CH})$, $133.5(\mathrm{CH}), 136.0(\mathrm{CH}), 141.7,147.0,151.4,152.3,158.1,159.4,168.1,188.5$. EIMZ $(\mathrm{m} / \mathrm{z}) \%=$ 506.2 (26), 465.1 (6), 433.1 (9), 393.1 (3), 337.1 (4), 329.2 (10), 271.1 (17), 230.1 (10), 230.1 
(12), 189.0 (17), 163.0 (13), 113.1 (46), 41.0 (100). HREIMS: $m / z$ 506.2309 (calcd. 506.2305 for $\left.\mathrm{C}_{30} \mathrm{H}_{34} \mathrm{O}_{7}\right)$.

4,6-Diallylphomosine A (4a): Colorless oil (22 mg; 18\%). after column chromatography purification [ $n$-hexane-EtOAc (7:1)]. IR $v_{\max }\left(\mathrm{CH}_{2} \mathrm{Cl}_{2}\right): 3330,2880,1650,1550,1280 \mathrm{~cm}^{-1} .{ }^{1} \mathrm{H}$ NMR (500 MHz, $\left.\mathrm{CDCl}_{3}\right): \delta=2.17,2.18$ and 2.32 (each $3 \mathrm{H}, \mathrm{s}$, Aryl-CH $\left.\mathrm{CH}_{3}\right), 3.95\left(\mathrm{~s}, 3 \mathrm{H}, \mathrm{OCH}_{3}\right)$, $4.63\left(\mathrm{dq}, J=5.0,1.2 \mathrm{~Hz}, 4 \mathrm{H}, 2 \times \mathrm{OCH}_{2} \mathrm{CH}=\mathrm{CH}_{2}\right), 5.13(\mathrm{dq}, J=10.0,1 \mathrm{H}, 1.5 \mathrm{~Hz}$, cis$\left.\mathrm{CH}_{2} \mathrm{CH}=\mathrm{CH}_{2}\right), 5.22\left(\mathrm{dq}, J=17.0,1.5,1 \mathrm{H}\right.$, trans $\left.-\mathrm{CH}_{2} \mathrm{CH}=\mathrm{CH}_{2}\right), 5.33(1 \mathrm{H}, \mathrm{dq}, J 10.0$ and 1.5 , cis $\left.-\mathrm{CH}_{2} \mathrm{CH}=\mathrm{CH}_{2}\right), 5.51\left(\mathrm{dq}, J=17.0\right.$ and $1.5 \mathrm{~Hz}, 1 \mathrm{H}$, trans $\left.-\mathrm{CH}_{2} \mathrm{CH}=\mathrm{CH}_{2}\right), 5.80\left(\mathrm{~s}, 1 \mathrm{H}, 6^{\prime}-\mathrm{H}\right)$, $5.90\left(\mathrm{~m}, 1 \mathrm{H}, \mathrm{CH}_{2} \mathrm{CH}=\mathrm{CH}_{2}\right), 6.08\left(\mathrm{~m}, 1 \mathrm{H}, \mathrm{CH}_{2} \mathrm{CH}=\mathrm{CH}_{2}\right), 6.38$ (s, $\left.1 \mathrm{H}, 4^{\prime}-\mathrm{H}\right), 10.68$ (s, $1 \mathrm{H}$, $\mathrm{CHO}), 11.64\left(\mathrm{~s}, 1 \mathrm{H}, 3^{\prime}-\mathrm{OH}\right) .{ }^{13} \mathrm{C} \mathrm{NMR}\left(125 \mathrm{MHz}, \mathrm{CDCl}_{3}\right): \delta=9.3,15.0,22.7\left(\mathrm{CH}_{3}\right), 52.2$ $\left(\mathrm{OCH}_{3}\right), 69.5$ and $74.6\left(2 \times \mathrm{OCH}_{2} \mathrm{CH}=\mathrm{CH}_{2}\right), 106.7(\mathrm{CH}), 106.8(\mathrm{CH}), 108.2,112.2,116.7,117.8$ $(\mathrm{CH}), 118.3(\mathrm{CH}), 118.8,131.4,131.8,132.4(\mathrm{CH}), 133.4(\mathrm{CH}), 138.2,147.3,154.9,159.8$, 160.2, 161.4, 172.2, 188.1. EIMS (m/z) \% = 426.2 (100), 394.1 (12), 353.1 (7), 312 (16), 297.1 (16), 237 (14), 191.1 55), 113.1 (43). HREIMS: $m / z 426.1674$ (calcd. 426.1679 for $\mathrm{C}_{24} \mathrm{H}_{26} \mathrm{O}_{7}$ ).

3',4,6-Triallylphomosine A (4b): A solution of phomosine A (1) (78 mg; $0.225 \mathrm{mmol})$ in dry dimethylformamide $(5 \mathrm{ml})$ was treated with potassium carbonate $(155 \mathrm{mg} ; 1.125 \mathrm{mmol})$ and allyl bromide (136 mg; $1.125 \mathrm{mmol} ; 0.097 \mathrm{ml}$ ) and the mixture was stirred under Ar for $18 \mathrm{~h}$ at $25^{\circ} \mathrm{C}$ after which water was added and the organic material was extracted with diethyl ether. The organic phase was dried $\left(\mathrm{Na}_{2} \mathrm{SO}_{4}\right)$ and evaporated under reduced pressure to afford a residue which was chromatographed using EtOAc-hexane (1:7) as eluent to give the triallyl ether $\mathbf{4 b}$ (66 mg; 63\%) as a white crystalline material, m.p. $130-131{ }^{\circ} \mathrm{C}$ (from $n$-hexane: $\mathrm{CH}_{2} \mathrm{Cl}_{2}$ ). IR $v_{\max }$ $\left(\mathrm{CH}_{2} \mathrm{Cl}_{2}\right): 2810,1650,1540,1285 \mathrm{~cm}^{-1} .{ }^{1} \mathrm{H}$ NMR (500 $\left.\mathrm{MHz}, \mathrm{CDCl}_{3}\right): \delta=2.07,2.20$ and 2.21 (each $3 \mathrm{H}, \mathrm{s}$, Aryl- $\left.\mathrm{CH}_{3}\right), 3.90\left(\mathrm{~s}, 3 \mathrm{H}, \mathrm{OCH}_{3}\right), 4.42$ (dq, $J=5.5,1.5 \mathrm{~Hz}, 4 \mathrm{H}, 2 \times \mathrm{OCH}_{2} \mathrm{CH}=\mathrm{CH}_{2}$ ), $4.63\left(\mathrm{dq}, J=5.5,1.5 \mathrm{~Hz}, 2 \mathrm{H}, \mathrm{OCH}_{2} \mathrm{CH}=\mathrm{CH}_{2}\right), 5.12(\mathrm{dq}, J=10.0,1.5 \mathrm{~Hz}, 1 \mathrm{H}$, cis$\left.\mathrm{CH}_{2} \mathrm{CH}=\mathrm{CH}_{2}\right), 5.21\left(\mathrm{dq}, J=17.0,1.5 \mathrm{~Hz}, 1 \mathrm{H}\right.$, trans $\left.-\mathrm{CH}_{2} \mathrm{CH}=\mathrm{CH}_{2}\right), 5.26(\mathrm{dq}, 1 \mathrm{H}, J=10.0,1.5$ $\left.\mathrm{Hz}, c i s-\mathrm{CH}_{2} \mathrm{CH}=\mathrm{CH}_{2}\right), 5.33\left(\mathrm{dq}, J=10.0,1.5 \mathrm{~Hz}, 1 \mathrm{H}\right.$, cis $\left.-\mathrm{CH}_{2} \mathrm{CH}=\mathrm{CH}_{2}\right), 5.40(\mathrm{dq}, J=17.0,1.5$ $\mathrm{Hz} 5,1 \mathrm{H}$, trans $\left.-\mathrm{CH}_{2} \mathrm{CH}=\mathrm{CH}_{2}\right), 5.51\left(\mathrm{dq}, J=17.0,1.5 \mathrm{~Hz}, 1 \mathrm{H}\right.$, trans $\left.-\mathrm{CH}_{2} \mathrm{CH}=\mathrm{CH}_{2}\right), 5.86(\mathrm{~s}, 1$ $\left.\mathrm{H}, 6^{\prime}-\mathrm{H}\right), 5.90\left(\mathrm{~m}, 1 \mathrm{H}, \mathrm{CH}_{2} \mathrm{CH}=\mathrm{CH}_{2}\right), 6.07$ (m, $\left.2 \mathrm{H}, 2 \times \mathrm{CH}_{2} \mathrm{CH}=\mathrm{CH}_{2}\right), 6.39$ (s, $\left.1 \mathrm{H}, 4^{\prime}-\mathrm{H}\right), 10.66$ (s, $1 \mathrm{H}, \mathrm{CHO}) .{ }^{13} \mathrm{C}$ NMR $\left(125 \mathrm{MHz}, \mathrm{CDCl}_{3}\right): \delta=10.1,13.0,22.7\left(\mathrm{CH}_{3}\right), 52.4\left(\mathrm{OCH}_{3}\right), 69.5,74.5$ $75.3\left(\mathrm{OCH}_{2} \mathrm{CH}=\mathrm{CH}_{2}\right), 106.8(\mathrm{CH}), 107.0(\mathrm{CH}), 112.2,117.3(\mathrm{CH}), 117.8(\mathrm{CH}), 118.2(\mathrm{CH})$, 124.7, 125.5, 127.9, $132.4(\mathrm{CH}), 133.4(\mathrm{CH}), 133.5(\mathrm{CH}), 141.7,147.4,151.5,152.3,159.8$, 161.3, 168.1, 188.0. EIMZ (m/z) \% = 466.1 (25), 425.1 (9), 393.1 (18), 353.1 (3), 311.1 (2), 279.1 (6), 191.1 (20), 149.0 (19), 81.1 (22). HREIMS: m/z 466.1987 (calcd. 466.1992 for $\mathrm{C}_{27} \mathrm{H}_{30} \mathrm{O}_{7}$ ).

Phomosine A oxime (5): A solution of phomosine A (1) (100 mg; $0.29 \mathrm{mmol}$ ) in ethanol (4 ml) was treated with hydroxylamine hydrochloride $(24 \mathrm{mg} ; 0.34 \mathrm{mmol})$ and stirred. The milky solution became clear after $6 \mathrm{~h}$ and after a further $8 \mathrm{~h}$ a dense white precipitate formed. Removal of the solvent and trituration of the residue with dichloromethane (DCM) (3 ml) afforded a white solid of the oxime (89 mg; 85\%), m.p. 241-242 ${ }^{\circ} \mathrm{C}$ (decomp.); IR $v_{\max }\left(\mathrm{CH}_{2} \mathrm{Cl}_{2}\right)$ : 3420, 2810, 1670, 1650, 1530, $1290 \mathrm{~cm}^{-1} .{ }^{1} \mathrm{H}$ NMR (500 MHz, $\left.\mathrm{CDCl}_{3} \backslash \mathrm{CD}_{3} \mathrm{OD}\right): \delta=2.01,2.03$ and 2.08 
(each $3 \mathrm{H}, \mathrm{s}$, Aryl-CH $\mathrm{CH}_{3}, 3.22$ (s, $\left.1 \mathrm{H}, \mathrm{NOH}\right), 3.79$ (s, $\left.3 \mathrm{H}, \mathrm{OCH}_{3}\right), 5.61$ (s, $\left.1 \mathrm{H}, 6^{\prime}-\mathrm{H}\right), 6.28$ (s, 1 $\left.\mathrm{H}, 4^{\prime}-\mathrm{H}\right)$ and 8.69 (s, $\left.1 \mathrm{H}, \mathrm{CH}=\mathrm{NOH}\right) .{ }^{13} \mathrm{C} \mathrm{NMR}\left(125 \mathrm{MHz}, \mathrm{CDCl}_{3} \backslash \mathrm{CD}_{3} \mathrm{OD}\right): \delta=7.95,14.9$, $21.6\left(\mathrm{CH}_{3}\right), 51.7\left(\mathrm{OCH}_{3}\right), 103.6,104.3(\mathrm{CH}), 104.4,110.6,110.8(\mathrm{CH}), 130.9,133.25,142.5$, 147.25, 153.1, 156.2, 158.3, 159.7 and 172.3EIMS $\left(180^{\circ} \mathrm{C}, 70 \mathrm{eV}\right): \mathrm{m} / z=362.08(8), 361.06$ (40), 344.08 (92), 312.04 (100), 284.08 (18), 256.07 (15), 180.04 (8), 151.04 (12). HREIMS: $\mathrm{m} / z$ 361.1157 (calcd. 361.1162 for $\mathrm{C}_{18} \mathrm{H}_{19} \mathrm{NO}_{6}$ ).

\section{4,6-Diacetyl phomosine A oxime (6a):}

Method A: A solution of triacetyl-phomosine A (2a) $(26 \mathrm{mg}$; $0.055 \mathrm{mmol}$ ) in ethanol (4 ml) was treated with hydroxylamine hydrochloride $(10 \mathrm{mg} ; 0.14 \mathrm{mmol})$ and the slightly yellow solution was stirred and monitored by TLC until all starting material was consumed $(6 \mathrm{~h})$. Evaporation of the solution afforded the diacetyl phomosine A oxime (2b) (26 mg; 97\%) as white feathery crystals.

\section{Method B:}

A solution of 4,6-diacetyl phomosine A $(\mathbf{2 b})(43 \mathrm{mg} ; 0.10 \mathrm{mmol})$ in ethanol $(4 \mathrm{ml})$ was treated with hydroxylamine hydrochloride $(10 \mathrm{mg} ; 0.14 \mathrm{mmol})$ and the milky solution was stirred for $6 \mathrm{~h}$ after which time it became clear and the reaction was complete (TLC monitoring). Evaporation of the solvent afforded the same oxime $\mathbf{6 a}$ derived from the triacetate described vide infra as a white solid (50 mg; 100\%), m.p. 210-211 ${ }^{\circ} \mathrm{C}$ (from EtOAc-hexane) and all spectral data are identical with the material synthesized above.

M.p. 212-214 ${ }^{\circ} \mathrm{C}$ (from EtOAc-hexane). IR $v_{\max }\left(\mathrm{CH}_{2} \mathrm{Cl}_{2}\right)$ : 3320, 2820, 1670, 1650, 1540, 1280 $\mathrm{cm}^{-1} .{ }^{1} \mathrm{H}$ NMR $\left(500 \mathrm{MHz}, \mathrm{CDCl}_{3}\right): \delta=1.98,2.04,2.18,2,19$ and 2.31 (each $3 \mathrm{H}$, s, Aryl-CH and $\mathrm{CH}_{3} \mathrm{CO}$ ), 3.91 (s, $\left.3 \mathrm{H}, \mathrm{OCH}_{3}\right), 5.80$ (s, $\left.1 \mathrm{H}, 6^{\prime}-\mathrm{H}\right), 6.48$ (s, $\left.1 \mathrm{H}, 4^{\prime}-\mathrm{H}\right), 7.19$ (s, $\left.1 \mathrm{H}, \mathrm{NOH}\right)$, 8.77 (s, $1 \mathrm{H}, \mathrm{CH}=\mathrm{NOH}), 10.10$ (bs, $\left.1 \mathrm{H}, 3^{\prime}-\mathrm{OH}\right) .{ }^{13} \mathrm{C}$ NMR $\left(125 \mathrm{MHz}, \mathrm{CDCl}_{3}\right): \delta=10.4,13.6$, 20.0, 20.4, $21.9\left(\mathrm{CH}_{3}\right.$ and $\left.\mathrm{CH}_{3} \mathrm{CO}\right), 52.4\left(\mathrm{OCH}_{3}\right), 103.3(\mathrm{CH}), 105.4,111.6(\mathrm{CH}), 124.7,125.7$, $129.5,142.7,143.4,144.1,144.4,148.3,155.8,158.6,166.2,167.4,168.4$. EIMS $(\mathrm{m} / z) \%=$ 445.1 848), 403.2 (22), 344.1 (100), 312.1 (84), 284.1 (7), 256.1 (8), 209.1 (6), 151.0 (7), 83.0 (14). HREIMS: $m / z 445.1372$ (calcd. 445.1373 for $\mathrm{C}_{22} \mathrm{H}_{23} \mathrm{NO}_{9}$ ).

4,6-Diacetyl-3'-methylphomosine A oxime methylether (6b): A solution of diacetyl phomosine A oxime (5) $(20 \mathrm{mg} ; 0.041 \mathrm{mmol})$ in acetone $(5 \mathrm{ml})$ was treated with methyl iodide (12 $\mathrm{mg} ; 0.05 \mathrm{ml} ; 0.082 \mathrm{mmol})$ and argentous oxide (19 $\mathrm{mg} ; 0.082 \mathrm{mmol})$; the mixture was rapidly stirred until the starting material was consumed (TLC monitoring). The solvent was removed under reduced pressure and the residue was chromatographed using EtOAc-hexane (3:7) as the eluent to afford the product methyl ether $\mathbf{6 b}(17 \mathrm{mg} ; \%)$ as white crystals m.p. 135$136^{\circ} \mathrm{C}^{1}{ }^{1} \mathrm{IR} \nu_{\max }\left(\mathrm{CH}_{2} \mathrm{Cl}_{2}\right): 2830,1665,1640,1530,1280 \mathrm{~cm}^{-1} ; \mathrm{H} \mathrm{NMR}\left(500 \mathrm{MHz}, \mathrm{CDCl}_{3}\right): \delta=$ 1.97, 2.07, 2.17, 2.23 and 2.30 (each $3 \mathrm{H}, \mathrm{s}, 3$ Aryl- $\mathrm{CH}_{3}$ and $2 \mathrm{xCH}_{3} \mathrm{CO}$ ), 3.87, 3.90 and 3.97 (each $\left.3 \mathrm{H}, \mathrm{s}, 3 \mathrm{OCH}_{3}\right), 5.92\left(\mathrm{~s}, 1 \mathrm{H}, 6^{\prime}-\mathrm{H}\right), 6.43\left(\mathrm{~s}, 1 \mathrm{H}, 4^{\prime}-\mathrm{H}\right), 8.48\left(\mathrm{~s}, 1 \mathrm{H}, \mathrm{CH}=\mathrm{NOCH}_{3}\right) .{ }^{13} \mathrm{C}$ NMR (125 MHz, $\left.\mathrm{CDCl}_{3}\right): \delta=10.4,13.6,20.2,20.4,22.1\left(\mathrm{CH}_{3}\right.$ and $\left.\mathrm{CH}_{3} \mathrm{CO}\right), 52.4\left(\mathrm{CO}_{2} \mathrm{CH}_{3}\right)$, $56.1($ Aryl-OCH 3$), 61.7\left(\mathrm{~N}_{3}-\mathrm{OCH}_{3}\right), 106.4(\mathrm{CH}), 106.8,107.2(\mathrm{CH}), 124.7,125.5,129.6,141.7$, 143.0, 143.2, 144.2, 156.4, 158.8, 166.3, 167.6, 168.4. EIMS $(m / z) \%=473.2(6), 442.2(4)$, 
400.2 (6), 346.1 (25), 314.1 (28), 286.1 (16), 258.1 (6), 191.1 (89), 151.1 (22), 125.1 (20). HREIMS: $m / z 473.1684$ (calcd. 473.1686 for $\mathrm{C}_{24} \mathrm{H}_{27} \mathrm{NO}_{9}$ ).

4,6-Diacetyl-3'-ethyl phomosine A oxime ethylether (6c): A solution of diacetyl phomosine A oxime (6a) (27 mg; $0.057 \mathrm{mmol})$ in acetone $(5 \mathrm{ml})$ was treated with ethyl iodide $(18 \mathrm{mg} ; 0.09$ $\mathrm{ml} ; 0.115 \mathrm{mmol}$ ) and argentous oxide (27 $\mathrm{mg} ; 0.115 \mathrm{mmol})$; the mixture was rapidly stirred until the starting material was consumed (TLC monitoring ). The solvent was removed under reduced pressure and the residue was chromatographed using EtOAc-hexane (3:7) as the eluent to afford the product 6a as an oil (27 mg; $94 \%$ ), which crystallized on standing to white crystals, m.p. 90$92{ }^{\circ} \mathrm{C}$; IR $v_{\max }\left(\mathrm{CH}_{2} \mathrm{Cl}_{2}\right): 2840,1660,1640,1530,1280 \mathrm{~cm}^{-1} .{ }^{1} \mathrm{H} \mathrm{NMR}\left(500 \mathrm{MHz}, \mathrm{CDCl}_{3}\right): \delta=$ 3.63 (t, $\left.3 \mathrm{H}, J=7.0 \mathrm{~Hz}, \mathrm{CH}_{2} \mathrm{CH}_{3}\right), 1.44\left(\mathrm{t}, J=7.0 \mathrm{~Hz}, 3 \mathrm{H}, \mathrm{CH}_{2} \mathrm{CH}_{3}\right), 1.97,2.07,2.16,2.21$, and 2.30 (each $3 \mathrm{H}, \mathrm{s}, 3$ Aryl- $\mathrm{CH}_{3}$ and $2 \mathrm{CH}_{3} \mathrm{CO}$ ), $3.90\left(\mathrm{~s}, 3 \mathrm{H}, \mathrm{OCH}_{3}\right), 4.06(\mathrm{q}, J=7.0 \mathrm{~Hz}, 2 \mathrm{H}$, $\left.\mathrm{CH}_{2} \mathrm{CH}_{3}\right), 4.20$ (q, J = 7.0 Hz, $\left.2 \mathrm{H}, \mathrm{CH}_{2} \mathrm{CH}_{3}\right), 5.90$ (s, $\left.1 \mathrm{H}, 6^{\prime}-\mathrm{H}\right), 6.40$ (s, $\left.1 \mathrm{H}, 4^{\prime}-\mathrm{H}\right), 8.47$ (s, 1 $\mathrm{H}, \mathrm{CH}=\mathrm{NOEt}) .{ }^{13} \mathrm{C} \mathrm{NMR}\left(125 \mathrm{MHz}, \mathrm{CDCl}_{3}\right): \delta=10.4,13.6,14.6,14.8,20.2,20.4,22.0\left(\mathrm{CH}_{3}\right.$ and $\left.\mathrm{CH}_{3} \mathrm{CO}\right), 52.3\left(\mathrm{OCH}_{3}\right), 64.6$ and $69.4\left(2 \times \mathrm{OCH}_{2} \mathrm{CH}_{3}\right), 107.1(\mathrm{CH}), 107.3,107.5(\mathrm{CH}), 124.6$, 125.5, 129.6, 141.3, 142.7, 143.1, 144.2, 144.3, 156.2, 158.3, 166.3, 167.6 and 168.4. EIMS $(\mathrm{m} / \mathrm{z}) \%=501.3$ (60), 414.2 (52), 372.1 (100), 311.1 (14), 253.1 (14), 208.1 (13), 149.0 (17). HREIMS: $m / z, 501.1998$ (calcd. 501.1999 for $\mathrm{C}_{26} \mathrm{H}_{31} \mathrm{NO}_{9}$ ).

Benzylation of phomosine A oximes: A solution of 4,6-diacetylphomosine A oxime (6a) (50 $\mathrm{mg} ; 0.103 \mathrm{mmol}$ ) in acetone $(5 \mathrm{ml})$ was treated with benzyl bromide $(40 \mathrm{mg} ; 0.234 \mathrm{mmol} ; 0.016$ $\mathrm{ml}$ ) and argentous oxide (57 $\mathrm{mg} ; 0.234 \mathrm{mmol})$ and the resulting mixture was stirred at $20{ }^{\circ} \mathrm{C}$ for $24 \mathrm{~h}$. The suspension was filtered, the solvent evaporated under reduced pressure and chromatogaphic separation of the residue afforded the following compounds in order of their elution using EtOAc-hexane (3:7) as eluent.

4,6-Diacetyl-3',4'-dibenzylphomosine A oxime benzylether (7): Yield $20 \mathrm{mg}$ (27\%) as white crystals, m.p. $128-129{ }^{\circ} \mathrm{C}$. IR $v_{\max }\left(\mathrm{CH}_{2} \mathrm{Cl}_{2}\right): 2850,1670,1640,1530,1280 \mathrm{~cm}^{-1} .{ }^{1} \mathrm{H}$ NMR $(500$ $\mathrm{MHz}, \mathrm{CDCl}_{3}$ ): $\delta=1.97,1.99,2.10,2.21,2.31$ (each $3 \mathrm{H}, \mathrm{s}, 3$ Aryl-CH 3 and $2 \mathrm{CH}_{3} \mathrm{CO}$ ), 3.91 (s, 3 $\left.\mathrm{H}, \mathrm{OCH}_{3}\right), 4.04\left(\mathrm{~s}, 2 \mathrm{H}, \operatorname{ArylCH}{ }_{2} \mathrm{Ph}\right), 4.77$ (s, $\left.2 \mathrm{H}, \operatorname{Aryl}-\mathrm{OCH}_{2} \mathrm{Ph}\right), 5.13\left(\mathrm{~s}, 2 \mathrm{H}, \mathrm{N}-\mathrm{OCH} \mathrm{Ph}_{2}\right.$, 6.17 (s, $\left.1 \mathrm{H}, 6^{\prime}-\mathrm{H}\right), 7.30$ (m, $\left.15 \mathrm{H}, 3 \mathrm{Ph}\right), 8.62$ (s, $\left.1 \mathrm{H}, \mathrm{CH}=\mathrm{NOCH}_{2} \mathrm{Ph}\right) .{ }^{13} \mathrm{C} \mathrm{NMR}(125 \mathrm{MHz}$, $\left.\mathrm{CDCl}_{3}\right): \delta=10.5$. 13.7, 20.1, 20.3, $20.4\left(\mathrm{CH}_{3}\right.$ and $\left.\mathrm{CH}_{3} \mathrm{CO}\right), 31.9\left(\mathrm{Aryl} \mathrm{CH}_{2} \mathrm{Ph}\right), 52.4\left(\mathrm{OCH}_{3}\right)$, 75.7 and $76.1\left(2 \mathrm{OCH}_{2} \mathrm{Ph}\right), 112.0,112.6,124.8,125.6,125.8,127.3,127.7(4 \times), 127.8(2 \times)$, $128.1(2 \times), 128.3(6 \times), 129.5,137.2,137.9,140.3,141.6,143.1,143.5,144.4,144.2,154.7$, 156.6, 166.3, 167.4 and 168.3. EIMS $(\mathrm{m} / \mathrm{z}) \%=715.3(4), 608.3(68), 519.2(51), 477(16), 401.2$ (15), 387.2 (9), 330.1 (26), 211.1 (16), 91.1 (100), 624 (8), 608 (16), 518 (8), 474 (10), 401 (12), 180 (8), 91 (100). HREIMS: $m / z 715.2786$ (calcd. 715.2781 for $\mathrm{C}_{43} \mathrm{H}_{41} \mathrm{NO}_{9}$ ).

4,6-Diacetyl-3'-benzylphomosine A oxime benzylether (6d): Yield $42 \mathrm{mg}(65 \%)$ as white crystals, m.p. $120-121^{\circ} \mathrm{C}$ (from EtOAc-hexane). IR $v_{\max }\left(\mathrm{CH}_{2} \mathrm{Cl}_{2}\right): 2820,1665,16451530,1280$ $\mathrm{cm}^{-1} .{ }^{1} \mathrm{H} \mathrm{NMR}\left(500 \mathrm{MHz}, \mathrm{CDCl}_{3}\right): \delta=1.97,2.00,2.15,2.22,2.31$ (each $3 \mathrm{H}, \mathrm{s}, 3 \times$ Aryl-CH 3 and $\left.2 \times \mathrm{CH}_{3} \mathrm{CO}\right), 3.90\left(\mathrm{~s}, 3 \mathrm{H}, \mathrm{OCH}_{3}\right), 5.13\left(\mathrm{~s}, 2 \mathrm{H}, \operatorname{ArylCH}_{2} \mathrm{Ph}\right), 5.21\left(\mathrm{~s}, 2 \mathrm{H}, \mathrm{N}-\mathrm{OCH}_{2} \mathrm{Ph}\right), 5.95(\mathrm{~s}, 1$ $\left.\mathrm{H}, 6^{\prime}-\mathrm{H}\right), 6.51\left(\mathrm{~s}, 1 \mathrm{H}, 4^{\prime}-\mathrm{H}\right), 7.35(\mathrm{~m}, 10 \mathrm{H}, 2 \times \mathrm{Ph}), 8.62$ (s, $\left.1 \mathrm{H}, \mathrm{CH}=\mathrm{NOCH}_{2} \mathrm{Ph}\right) .{ }^{13} \mathrm{C} \mathrm{NMR}$ (125 MHz, $\left.\mathrm{CDCl}_{3}\right): \delta=10.4,13.6,20.2,20.4,22.1\left(\mathrm{CH}_{3}\right.$ and $\left.\mathrm{CH}_{3} \mathrm{CO}\right), 52.4\left(\mathrm{OCH}_{3}\right), 70.7$ and 
$76.1\left(2 \times \mathrm{OCH}_{2} \mathrm{Ph}\right), 107.5,107.6,107.9,124.7,125.5,127.2$ (2), 127.7 (2), 127.8 (2), 128.3, 128.4 (2), 128.5 (2), 129.6, 136.8, 138.1, 141.7, 143.0, 143.4, 144.2, 156.5, 157.9, 166.3, 167.6, 168.4. EIMS $(\mathrm{m} / \mathrm{z}) \%=625(9), 582.2$ (2), 518.2 (9), 471.1 (7), 429.1 (10), 343.1 (28), 311.1 (54), 279.2 (12), 180.0 (14), 149.0 (38), 91.1 (100). HREIMS: $m / z 625.2313$ (calcd. 625.2312 for $\mathrm{C}_{36} \mathrm{H}_{35} \mathrm{NO}_{9}$ ).

$N$-Methoxyphomosine A imine (8a): To a solution of phomosine A (1) (100 mg; $0.29 \mathrm{mmol})$ in EtOH (4 ml) was added methoxyamine $(29 \mathrm{mg}$; $0.35 \mathrm{mmol})$. The solution became clearer during the stirring and later a dense white precipitate formed. After $3 \mathrm{~h}$ the solvent was removed and the residue taken up in DCM and washed with water. The solvent was removed under reduced pressure to give a final product, which was purified using chromatography, and the eluent EtOAc-hexane (3:7) to afford the methoxyimine (90 mg; 83\%) as white crystals, m.p. 213-214 ${ }^{\circ} \mathrm{C}$. IR $v_{\max }\left(\mathrm{CH}_{2} \mathrm{Cl}_{2}\right): 3330,2825,1660,16301530,1280 \mathrm{~cm}^{-1} .{ }^{1} \mathrm{H}$ NMR $\left(500 \mathrm{MHz}, \mathrm{CDCl}_{3}\right): \delta$ = 2.16, 2.18, 2.21 (each $3 \mathrm{H}, \mathrm{s}, 3 \times$ Aryl- $\left.\mathrm{CH}_{3}\right), 3.92\left(\mathrm{~s}, 3 \mathrm{H}, \mathrm{CO}_{2} \mathrm{CH}_{3}\right), 4.01$ (s, $\left.3 \mathrm{H}, \mathrm{N}-\mathrm{OCH}_{3}\right)$, 5.74 (s, $\left.1 \mathrm{H}, 6^{\prime}-\mathrm{H}\right), 5.76$ (s, $\left.1 \mathrm{H}, 4-\mathrm{OH}\right), 6.49$ (s, $\left.1 \mathrm{H}, 4^{\prime}-\mathrm{H}\right), 8.80$ (s, $\left.1 \mathrm{H}, \mathrm{CH}=\mathrm{NOCH}_{3}\right), 10.26$ (s, $1 \mathrm{H}, 6-\mathrm{OH}), 11.85$ (s, $\left.1 \mathrm{H}, 3^{\prime}-\mathrm{OH}\right) .{ }^{13} \mathrm{C} \mathrm{NMR}\left(125 \mathrm{MHz}, \mathrm{CDCl}_{3}\right): \delta=8.2,15.3,22.0\left(\mathrm{CH}_{3}\right), 52.0$ $\left(\mathrm{CO}_{2} \mathrm{CH}_{3}\right), 62.5\left(\mathrm{~N}-\mathrm{OCH}_{3}\right), 103,3,104.4,105.0,110.5,111.9,130.8,132.7,143.3,146.5,152.2$, 155.9, 159.0, 160.4 and 172.3. EIMS $m / z(5)=375.06(10), 344$ (100), 312 (94), 284 (18), 256 (18), 227 (8), 171 (7), 83 (13). HREIMS: $m / z 375.1317$ (calcd. 375.1318 for $\mathrm{C}_{19} \mathrm{H}_{21} \mathrm{NO}_{7}$ ).

3',4,6-Triacetyl- $N$-methoxyphomosine $\mathrm{A}$ imine (8c): To a solution of triacetyl phomosine A (2a) $(15 \mathrm{mg}$. $0.032 \mathrm{mmol})$ in $\mathrm{EtOH}(3 \mathrm{ml})$ was added methoxyamine $(8 \mathrm{mg} .0 .09 \mathrm{mmol})$ and after a period of $3 \mathrm{~h}$ was worked up as described above to afford the product $2 \mathbf{a}(17 \mathrm{mg} ; 100 \%)$ as white crystals m.p. $145-146{ }^{\circ} \mathrm{C}$. IR $v_{\max }\left(\mathrm{CH}_{2} \mathrm{Cl}_{2}\right)$ : 1660, $16201540,1290 \mathrm{~cm}^{-1}$. ${ }^{1} \mathrm{H}$ NMR (500 $\mathrm{MHz}, \mathrm{CDCl}_{3}$ ): $\delta=1.95,2.02,2.20,2.24,2.30$ and 2.32 (each $3 \mathrm{H}, \mathrm{s}, 3 \times \mathrm{Aryl}-\mathrm{CH}_{3}$ and $3 \times \mathrm{CH}_{3} \mathrm{CO}$ ), 3.90 (s, $3 \mathrm{H}, \mathrm{CO}_{2} \mathrm{CH}_{3}$ ), 3.95 (s, $\left.3 \mathrm{H}, \mathrm{NOCH}_{3}\right), 6.21$ (s, $\left.1 \mathrm{H}, 6^{\prime}-\mathrm{H}\right), 6.60$ (s, $1 \mathrm{H}, 4^{\prime}-$ $\mathrm{H}), 8.45\left(\mathrm{~s}, 1 \mathrm{H}, \mathrm{CH}=\mathrm{NOCH}_{3}\right) .{ }^{13} \mathrm{C} \mathrm{NMR}\left(125 \mathrm{MHz}, \mathrm{CDCl}_{3}\right): \delta=10.4,13.6,20.0,20.4,21.2$, $21.5\left(\mathrm{CH}_{3}\right.$ and $\left.\mathrm{CH}_{3} \mathrm{CO}\right), 52.4\left(\mathrm{CO}_{2} \mathrm{CH}_{3}\right), 61.9\left(\mathrm{~N}-\mathrm{OCH}_{3}\right), 111.5,112.5,118.5,125.0,125.7$, $129.5,141.9,142.9,143.8,144.5,148.8,156.3,166.2,167.5,168.3$ and 169.4. EIMS $(\mathrm{m} / z) \%=$ 501.2 (27), 459.1 (26), 428.1 (27), 386.1 (58), 344.1 (78), 312.1 (58), 284.1 (8), 256.1 (12), 216.1 (6), 151.1 (14), 149 (20), 83 (50). HREIMS: m/z 501.1633 (calcd. 501.1635 for $\mathrm{C}_{25} \mathrm{H}_{27} \mathrm{NO}_{10}$ ).

4,6-Diacetyl- $N$-methoxyphomosine A imine (8b): To a warmed solution of 4,6diacetylphomosine (2b) $(95 \mathrm{mg} ; 0.22 \mathrm{mmol})$ in $\mathrm{EtOH}(5 \mathrm{ml})$ was added methoxyamine $(28 \mathrm{mg}$; $0.33 \mathrm{mmol}$ ); after stirring $1 \mathrm{~min}$ a white crystalline mass formed. After $0.5 \mathrm{~h}$ the reaction mixture was evaporated under reduced pressure to a solid mass which was dissolved in DCM (50 ml) and washed with water. The solution was dried $\left(\mathrm{Na}_{2} \mathrm{SO}_{4}\right)$, evaporated under reduced pressure to afford the methoxyimine $(102 \mathrm{mg} ; 100 \%)$ as white needles, m.p. 167-168. IR $v_{\max }\left(\mathrm{CH}_{2} \mathrm{Cl}_{2}\right)$ : 3340, 2830, 1650, 1620 1540, $1280 \mathrm{~cm}^{-1} .{ }^{1} \mathrm{H} \mathrm{NMR}$ (500 MHz, $\mathrm{CDCl}_{3}$ ): $\delta=2.00,2.06,2.19$, 2.21 and 2.31 (each $3 \mathrm{H}, \mathrm{s}, 3 \times$ Aryl $-\mathrm{CH}_{3}$ and $2 \times \mathrm{CH}_{3} \mathrm{CO}$ ), $3.93\left(\mathrm{~s}, 3 \mathrm{H}, \mathrm{CO}_{2} \mathrm{CH}_{3}\right.$ ), 4.01 (s, $3 \mathrm{H}$, $\left.\mathrm{NOCH}_{3}\right), 5.81$ (s, $\left.1 \mathrm{H}, 6^{\prime}-\mathrm{H}\right), 6.51$ (s, $\left.1 \mathrm{H}, 4^{\prime}-\mathrm{H}\right), 8.72$ (s, $\left.1 \mathrm{H}, \mathrm{CH}=\mathrm{NOCH}_{3}\right), 10.27$ (s, $1 \mathrm{H}, 3^{\prime}-$ $\mathrm{OH}) .{ }^{13} \mathrm{C}$ NMR $\left(125 \mathrm{MHz}, \mathrm{CDCl}_{3}\right): \delta=10.4,13.5,20.0,20.4,21.9\left(\mathrm{CH}_{3}\right.$ and $\left.\mathrm{CH}_{3} \mathrm{CO}\right), 52.4$ 
$\left(\mathrm{CO}_{2} \mathrm{CH}_{3}\right), 62.5\left(\mathrm{NOCH}_{3}\right), 103.3,105.3,111.6,124.7,125.7,129.5,142.6,143.2,144.1,144.4$, 146.6, 155.7, 158.7, 166.2, 167.4, 168.3. EIMS ( $\mathrm{m} / \mathrm{z}) \%=459.0$ (20), 417.0 (44), 386.0 (12), 344.1 (98), 312.1 (100), 284.1 (13), 256.0 (11), 216.0 (6), 167.1 (12), 149 (18). HREIMS: $\mathrm{m} / z$ 459.1531 (calcd. 459.1529 for $\mathrm{C}_{23} \mathrm{H}_{25} \mathrm{NO}_{9}$ ).

Methyl phomosine A hydrazonecarboxylate (9a): To a suspension of phomosine A (1) (110 $\mathrm{mg} ; 0.32 \mathrm{mmol})$ in EtOH $(5 \mathrm{ml})$ was added methyl hydrazinecarboxylate (40 mg; $0.44 \mathrm{mmol})$ and the resulting mixture was stirred under Ar for $1 \mathrm{~h}$ and the solid filtered off (44 $\mathrm{mg}$ ).. This was not investigated further due to its insolubility in all organic solvents. Evaporation of the mother liquor afforded the hydrazonecarboxylate (9a) $(85 \mathrm{mg} ; 64 \%)$ as feathery white crystals, m.p. 266-267 ${ }^{\circ} \mathrm{C}$. IR $v_{\max }\left(\mathrm{CH}_{2} \mathrm{Cl}_{2}\right): 3440,2830,1650,16201530,1280 \mathrm{~cm}^{-1} .{ }^{1} \mathrm{H}$ NMR $(500 \mathrm{MHz}$, DMSO- $d_{6}$ ): $\delta=2.06,2.11$ and 2.12 (each $3 \mathrm{H}, \mathrm{s}, 3 \times$ Aryl- $\left.\mathrm{CH}_{3}\right), 3.73\left(\mathrm{~s}, 3 \mathrm{H}, \mathrm{NHCO}_{2} \mathrm{CH}_{3}\right), 3.86$ (s, $3 \mathrm{H}, \mathrm{OCH}_{3}$ ), 5.66 (s, $\left.1 \mathrm{H}, 6^{\prime}-\mathrm{H}\right), 6.39$ (s, $\left.1 \mathrm{H}, 4^{\prime}-\mathrm{H}\right), 8.75$ (s, $\left.1 \mathrm{H}, 4-\mathrm{OH}\right), 9.70$ (s, $\left.1 \mathrm{H}, \mathrm{NH}\right)$, $11.00(\mathrm{~s}, 1 \mathrm{H}, \mathrm{CH}=\mathrm{N}), 11.40(\mathrm{~s}, 1 \mathrm{H}, 6-\mathrm{OH}), 11.80\left(\mathrm{~s}, 1 \mathrm{H}, 3^{\prime}-\mathrm{OH}\right) .{ }^{13} \mathrm{C} \mathrm{NMR}(125 \mathrm{MHz}$, DMSO-d $\left.d_{6}\right): \delta=9.3,14.9,22.0\left(\mathrm{CH}_{3}\right), 40.5\left(\mathrm{NOCH}_{3}\right), 52.6\left(\mathrm{OCH}_{3}\right), 104.0,105.4,106.7,111.0$, $111.1,130.0,133.7,137.6,142.4,153.2,154.1$. 157.1. 157.5, 159.3, 171.3. EIMS m/z $(5)=$ 418.1 (96), 386.1 (48), 344 (36), 241 (18), 210.1 (20), 151.0 (21), 83.0 (34). HREIMS: $\mathrm{m} / z$ 418.1375 (calcd. 418.1376 for $\mathrm{C}_{20} \mathrm{H}_{22} \mathrm{~N}_{2} \mathrm{O}_{8}$ ).

$N^{\prime}, N^{\prime}$-Dimethylphomosine A hydrazone (9b): To a stirred suspension of phomosine A (1) (100 $\mathrm{mg} ; 0.29 \mathrm{mmol})$ in EtOH $(5 \mathrm{ml})$ was added $N, N$-dimethylhydrazine $(19 \mathrm{mg} ; 0.38 \mathrm{mmol} ; 0.022$ $\mathrm{ml})$ under Ar to form a white precipitate of the hydrazone $(100 \mathrm{mg} ; 89 \%)$ with m.p. $227-228{ }^{\circ} \mathrm{C}$. IR $v_{\max }\left(\mathrm{CH}_{2} \mathrm{Cl}_{2}\right): 3430,2825,1650,16251530,1280 \mathrm{~cm}^{-1} .{ }^{1} \mathrm{H}$ NMR $\left(500 \mathrm{MHz}, \mathrm{CDCl}_{3}\right): \delta=$ 2.14, 2.19 and 2.23 (each $3 \mathrm{H}, \mathrm{s}, 3$ Aryl- $\left.\mathrm{CH}_{3}\right), 2.96$ [s, $6 \mathrm{H}, \mathrm{N}\left(\mathrm{CH}_{3}\right)_{2}$ ], $3.92\left(\mathrm{~s}, 3 \mathrm{H}, \mathrm{OCH}_{3}\right), 5.73$ (s, $\left.1 \mathrm{H}, 6^{\prime}-\mathrm{H}\right), 5.88$ (s, $\left.1 \mathrm{H}, 4-\mathrm{OH}\right), 6.44$ (s, $\left.1 \mathrm{H}, 4^{\prime}-\mathrm{H}\right), 7.98$ (s, $\left.1 \mathrm{H}, \mathrm{CH}=\mathrm{N}\right), 11.84$ (s, $1 \mathrm{H}, 6-$ $\mathrm{OH}), 12.10\left(\mathrm{~s}, 1 \mathrm{H}, 33^{\prime}-\mathrm{OH}\right) .{ }^{13} \mathrm{C} \mathrm{NMR}\left(125 \mathrm{MHz}, \mathrm{CDCl}_{3}\right): \delta=8.3,15.3$ and $21.8\left(\right.$ Aryl- $\left.\mathrm{CH}_{3}\right)$, $43.0\left[\mathrm{~N}\left(\mathrm{CH}_{3}\right)_{2}\right], 52.0\left(\mathrm{OCH}_{3}\right), 103.9,104.9,106.4,110.4,111.9,130.9,132.5,133.1,140.3$, 152.4, 154.8, 158.7, 160.3, 172.4. EIMS m/z (\%): 388.10 (100), 344.04 (73), 312 (90), 297 (38), 273 (14), 149 (7). HREIMS: $m / z 388.1633$ (calcd. 388.1634 for $\mathrm{C}_{20} \mathrm{H}_{24} \mathrm{~N}_{2} \mathrm{O}_{6}$ ).

$\boldsymbol{N}$-Propylphomosine A imine (9c): To a stirred suspension of phomosine A (1) (110 mg; 0.32 $\mathrm{mmol})$ in EtOH (5 ml) under Ar was added propylamine $(22.5 \mathrm{mg}$; $0.38 \mathrm{mmol}$; $0.031 \mathrm{ml})$; the yellow solution was stirred for $2 \mathrm{~h}$ and then the yellow crystalline mass was filtered off to give the imine (118 mg; 95\%) as yellow crystals m.p. 206-207 ${ }^{\circ} \mathrm{C}$. IR $\nu_{\max }\left(\mathrm{CH}_{2} \mathrm{Cl}_{2}\right): 3430,2835$, 1650, $16251530,1280 \mathrm{~cm}^{-1} .{ }^{1} \mathrm{H}$ NMR $\left(500 \mathrm{MHz}, \mathrm{CDCl}_{3}\right): \delta=1.01(\mathrm{t}, J=7.5 \mathrm{~Hz}, 3 \mathrm{H}$, $\mathrm{CH}_{2} \mathrm{CH}_{2} \mathrm{CH}_{3}$ ), 1.75, (sextet, $J=7.5 \mathrm{~Hz}, 2 \mathrm{H}, \mathrm{CH}_{2} \mathrm{CH}_{2} \mathrm{CH}_{3}$ ), 2.13, 2.20 and 2.24 (each $3 \mathrm{H}$, s, $3 \times$ Aryl- $\left.\mathrm{CH}_{3}\right), 3.56\left(\mathrm{t}, \mathrm{J}=7.5 \mathrm{~Hz}, 2 \mathrm{H}, \mathrm{CH}_{2} \mathrm{CH}_{2} \mathrm{CH}_{3}\right), 3.93\left(\mathrm{~s}, 3 \mathrm{H}, \mathrm{OCH}_{3}\right), 5.57\left(\mathrm{~s}, 1 \mathrm{H}, 6^{\prime}-\mathrm{H}\right)$, 6.39 (s, $\left.1 \mathrm{H}, 4^{\prime}-\mathrm{H}\right), 8.86$ (s, $\left.1 \mathrm{H}, \mathrm{CH}=\mathrm{N}\right), 11.85$ (s, $\left.1 \mathrm{H}, 3^{\prime}-\mathrm{OH}\right) .{ }^{13} \mathrm{C}$ NMR $\left(125 \mathrm{MHz}, \mathrm{CDCl}_{3}\right): \delta$ $=8.3(\mathrm{ArylCH}), 11.6\left(\mathrm{CH}_{2} \mathrm{CH}_{2} \mathrm{CH}_{3}\right), 15.3$ and 22. $3(\mathrm{ArylCH}), 24.1\left(\mathrm{CH}_{2} \mathrm{CH}_{2} \mathrm{CH}_{3}\right), 52.0$ $\left(\mathrm{OCH}_{3}\right), 59.4\left(\mathrm{CH}_{2} \mathrm{CH}_{2} \mathrm{CH}_{3}\right), 101.6,104.9,105.3,110.5,113.6,130.9,132.7,145.5,152.4$, 157.0, 159.2, 160.4, 167.1, 172.4. EIMS $m / z(\%)=387.0$ (86), 345.9 (82), 327.0 (100), 285.0 (8), 256.0 (8), 177.0 (15), 148.9 (25), 83 (37). HREIMS: $m / z 387.1681$ (calcd. 387.1682 for $\mathrm{C}_{21} \mathrm{H}_{25} \mathrm{NO}_{6}$ ). 
$\boldsymbol{N}$-Benzylphomosine imine (9d): To a stirred suspension of phomosine A (1) (102 mg; 0.29 $\mathrm{mmol}$ ) in EtOH $(5 \mathrm{ml})$ under Ar was added benzylamine (40 mg; $0.38 \mathrm{mmol} ; 0.04 \mathrm{ml})$; the resulting solution was stirred for $2 \mathrm{~h}$ after which the solvent was removed under vacuum. This afforded the imine $(117 \mathrm{mg} ; 93 \%)$ as yellow crystals, m.p. $197-198^{\circ} \mathrm{C}$. IR $v_{\max }\left(\mathrm{CH}_{2} \mathrm{Cl}_{2}\right): 3420$, 2825, 1650, 1620 1530, $1280 \mathrm{~cm}^{-1}$. ${ }^{1} \mathrm{H} \mathrm{NMR} \mathrm{(500} \mathrm{MHz,} \mathrm{CDCl}_{3}$ ): $\delta=2.15,2.20$ and 2.25 (each 3 $\mathrm{H}, \mathrm{s}, 3$ Aryl- $\left.\mathrm{CH}_{3}\right), 3.93\left(\mathrm{~s}, 3 \mathrm{H}, \mathrm{OCH}_{3}\right), 4.83$ (s, $\left.2 \mathrm{H}, \mathrm{CH}=\mathrm{NCH}_{2} \mathrm{Ph}\right), 5.66$ (s, $\left.1 \mathrm{H}, 6^{\prime}-\mathrm{H}\right), 6.44$ (s, $\left.1 \mathrm{H}, 4^{\prime}-\mathrm{H}\right), 7.35$ (m, $\left.5 \mathrm{H}, \mathrm{CH}_{2} \mathrm{Ph}\right), 9.07$ (s, $\left.1 \mathrm{H}, \mathrm{CH}=\mathrm{N}\right), 11.86$ (s, $\left.1 \mathrm{H}, 3^{\prime}-\mathrm{OH}\right) .{ }^{13} \mathrm{C}$ NMR (125 $\left.\mathrm{MHz}, \mathrm{CDCl}_{3}\right): \delta=8.3,15.3$ and $22.3\left(\mathrm{CH}_{3}\right), 52.0\left(\mathrm{OCH}_{3}\right), 62.7\left(\mathrm{CH}_{2} \mathrm{Ph}\right), 102.7,105.0,105.7$, 110.5, 112.8, 127.4, $127.6(2 \times), 128.7$ (2×), 130.9, 132.7, 138.2, 145.3, 152.4, 157.0, $160.4(2 \times)$, 164.6 and 172.3. EIMS $(\mathrm{m} / \mathrm{z}) \%=435.2$ (100), 403.2 (41), 344.1 (51), 312.1 (58), 284.1 (15), 225.1 (8), 149.0 (6), 91.0 (78). HREIMS: $m / z, 435.1682$ (calcd. 435.1682 for $\mathrm{C}_{25} \mathrm{H}_{25} \mathrm{NO}_{6}$ ).

Phomosine A-2'-alcohol (10a): A solution of phomosine A (1) (100 mg; $0.29 \mathrm{mmol}$ ) in ethanol $(5 \mathrm{ml})$ was stirred at $0^{\circ} \mathrm{C}$ and sodium borohydride $(44 \mathrm{mg} ; 1.16 \mathrm{mmol}$ ) was added at once. After slow gas evolution, the solution was stirred for a further $20 \mathrm{~min}$, the solvent was removed and the residue dissolved in EtOAc and washed with water. The dried $\left(\mathrm{Na}_{2} \mathrm{SO}_{4}\right)$ extract was evaporated to dryness and afforded the expected primary alcohol 10a as a white crystalline powder (100 mg; 100\%) m.p. $305{ }^{\circ} \mathrm{C}$. IR $v_{\max }\left(\mathrm{CH}_{2} \mathrm{Cl}_{2}\right): 3450,2825,1640,1530,1280 \mathrm{~cm}^{-1} .{ }^{1} \mathrm{H}$ NMR (500 MHz, $\mathrm{CD}_{3} \mathrm{CN}$ ): $\delta=2.06,2.13,2.41$ (each $\left.3 \mathrm{H}, \mathrm{s}, \operatorname{Aryl}-\mathrm{CH}_{3}\right), 3.95\left(\mathrm{~s}, 3 \mathrm{H}, \mathrm{OCH}_{3}\right.$ ), 4.10 (s, $\left.1 \mathrm{H}, \mathrm{CH}_{2} \mathrm{OH}\right), 4.91$ (s, $\left.2 \mathrm{H}, \mathrm{CH}_{2} \mathrm{OH}\right), 5.89$ (s, $\left.1 \mathrm{H}, 6^{\prime}-\mathrm{H}\right), 6.41$ (s, $\left.1 \mathrm{H}, 4^{\prime}-\mathrm{H}\right), 7.58$ (s, 1 $\mathrm{H}, 4-\mathrm{OH}), 9.02$ (s, $1 \mathrm{H}, 6-\mathrm{OH}), 11.81\left(\mathrm{~s}, 1 \mathrm{H}, 3^{\prime}-\mathrm{OH}\right) .{ }^{13} \mathrm{C} \mathrm{NMR}\left(125 \mathrm{MHz}, \mathrm{CD}_{3} \mathrm{CN}\right): \delta=7.4$, 14.7, $20.5\left(\mathrm{CH}_{3}\right), 51.7$ and $53.9\left(\mathrm{CH}_{2} \mathrm{OH}\right.$ and $\left.\mathrm{OCH}_{3}\right), 103.9,106.3(\mathrm{CH}), 110.3,110.8(\mathrm{CH})$, $112.5,131.7,134.64,139.8,153.6,155.7,157.3,159.8,172.5$. EIMS $\left(180{ }^{\circ} \mathrm{C}, 70 \mathrm{eV}\right): \mathrm{m} / z=$ 348.08 (12), 331.09 (12), 330.09 (70), 298.06 (100), 283.02 (29), 212.00 (12), 180.02 (13), 148.04 (14). HREIMS: $m / z, 348.1215$ (calcd. 348.1209 for $\mathrm{C}_{18} \mathrm{H}_{20} \mathrm{O}_{7}$ ).

4,6-Diacetylphomosine-A-2'-alcohol (10b): A suspension of phomosine A diacetate (2b) (83 $\mathrm{mg} ; 0.193 \mathrm{mmol})$ suspended in $\mathrm{EtOH}(6 \mathrm{ml})$ at $0^{\circ} \mathrm{C}$ was treated at once with sodium borohydride $(8.7 \mathrm{mg} ; 0.232 \mathrm{mmol}$ ) and after $5 \mathrm{~min}$ the solution started to clear. After a further 5 min sufficient $0.1 \mathrm{M}$ aqueous hydrogen chloride was added to destroy the excess of hydride after which water $(50 \mathrm{ml})$ was added and the product extracted into DCM. Removal of the solvent afforded the diacetylbenzyl alcohol (10b) (83 mg; 100\%) as white crystals m.p. $160-161{ }^{\circ} \mathrm{C}$. IR $v_{\max }\left(\mathrm{CH}_{2} \mathrm{Cl}_{2}\right): 3320,2830,1645,1530,1285 \mathrm{~cm}^{-1} .{ }^{1} \mathrm{H}$ NMR $\left(500 \mathrm{MHz}, \mathrm{CDCl}_{3}\right): \delta=1.97,2.05$, 2.15, 2.16, 2.30 (each $3 \mathrm{H}, \mathrm{s}, 3 \times \mathrm{Aryl}-\mathrm{CH}_{3}$ and $2 \times \mathrm{CH}_{3} \mathrm{CO}$ ), $3.90\left(\mathrm{~s}, 3 \mathrm{H}, \mathrm{OCH}_{3}\right), 5.08(\mathrm{~s}, 2 \mathrm{H}$, Aryl- $\left.\mathrm{CH}_{2} \mathrm{OH}\right), 5.80\left(\mathrm{~s}, 1 \mathrm{H}, 6^{\prime}-\mathrm{H}\right), 6.40$ (s, $\left.1 \mathrm{H}, 4^{\prime}-\mathrm{H}\right), 7.65$ (s, $\left.1 \mathrm{H}, \mathrm{OH}\right) .{ }^{13} \mathrm{C} \mathrm{NMR}(125 \mathrm{MHz}$, $\left.\mathrm{CDCl}_{3}\right): \delta=10.4,13.6,20.0,20.4,21.4\left(\mathrm{CH}_{3}\right.$ and $\left.\mathrm{CH}_{3} \mathrm{CO}\right), 52.4\left(\mathrm{OCH}_{3}\right), 58.1\left(\mathrm{ArylCH} \mathrm{H}_{2} \mathrm{OH}\right)$, 106.3, $109.8(\mathrm{CH}), 111.7(\mathrm{CH}), 124.5,125.6,129.4,139.9,142.9,143.9,144.1,154.3,157.1$, 166.3, 167.7, 168.4. EIMS $m / z(\%)=432.1$ (14), 399.1 (8), 372.1 (8), 330.1 (58), 298.1 (100), 241 (5), 199.1 (7), 151.0 (12), 137 (22). HREIMS: $m / z, 432.1422$ (calcd. 432.1420 for $\mathrm{C}_{22} \mathrm{H}_{24} \mathrm{O}_{9}$ ). Methyl 2,4-diacetoxy-5-(3-(benzyloxy)-2-(hydroxymethyl)-5-methylphenoxy)-3,6-dimethylbenzoate (11a): A solution of 4,6-diacetylphomosine A benzyl alcohol (10b) (60 mg; 0.139 
mmol) in acetone $(6 \mathrm{ml})$ was treated with benzyl bromide $(29 \mathrm{mg} ; 0.170 \mathrm{mmol} ; 0.02 \mathrm{ml})$ and argentous oxide (39 $\mathrm{mg}$; $0.17 \mathrm{mmol}$ ); the resulting mixture was stirred under Ar for $3 \mathrm{~h}$ and then chromatographed using EtOAc-hexane (3:7) as eluent to afford the benzyl ether (11a) (52 mg; $72 \%)$ as an oil. IR $v_{\max }\left(\mathrm{CH}_{2} \mathrm{Cl}_{2}\right): 3230,2855,1640,1530,1270 \mathrm{~cm}^{-1}$. ${ }^{1} \mathrm{H}$ NMR (500 MHz, $\mathrm{CDCl}_{3}$ ): $\delta=1.99,2.06,2.18,2.21$ and 2.30 (each $3 \mathrm{H}, \mathrm{s}, 3 \times$ Aryl- $\mathrm{CH}_{3}$ and $2 \times \mathrm{CH}_{3} \mathrm{CO}$ ), 3.90 (s, 3 $\left.\mathrm{H}, \mathrm{OCH}_{3}\right), 4.91\left(\mathrm{~s}, 2 \mathrm{H}\right.$, phenol- $\left.\mathrm{CH}_{2} \mathrm{OCH}_{2} \mathrm{Ph}\right), 5.11$ (s, $2 \mathrm{H}, \mathrm{PhCH}_{2} \mathrm{OCH}_{2}$-phenol), 5.92 (s, $1 \mathrm{H}$, H-6'), 6.51 (s, $\left.1 \mathrm{H}, \mathrm{H}-4^{\prime}\right), 7.40$ (m, $\left.5 \mathrm{H}, \mathrm{CH}_{2} P h\right) .{ }^{13} \mathrm{C} \mathrm{NMR}\left(125 \mathrm{MHz}, \mathrm{CDCl}_{3}\right): \delta=10.4,13.6$, 20.1, 20.4 and 21.9 (3×Aryl- $\mathrm{CH}_{3}$ and $\left.2 \times \mathrm{CH}_{3} \mathrm{CO}\right), 52.4\left(\mathrm{OCH}_{3}\right), 54.7$ (phenol- $\left.\mathrm{CH}_{2} \mathrm{OCH}_{2} \mathrm{Ph}\right), 70.6$ ( $\mathrm{PhCH}_{2} \mathrm{OCH}_{2}$-phenol), 107.1, 107.5, 114.8, 124.6, 125.6, 127.4 (2×), 128.2, 128.5 (2×), 129.7, 136.8, 139.8, 142.8, 144.2, 144.3, 155.7, 157.7, 166.3, 167.8 and 168.4. HREIMS: $m / z$ 522.1894 (calcd. 522.1890 for $\mathrm{C}_{29} \mathrm{H}_{30} \mathrm{O}_{9}$ ).

Methyl 3-(3-(benzyloxy)-2-(hydroxymethyl)-5-methylphenoxy)-4,6-dihydroxy-2,5-dimethylbenzoate (11b): To a solution of the diacetylbenzylether (11a) $(20 \mathrm{mg}$; $0.038 \mathrm{mmol})$ in $\mathrm{MeOH}$ $(4 \mathrm{ml})$ was added a few drops of sodium methoxide in $\mathrm{MeOH}$ [from sodium, $30 \mathrm{mg}$ in $\mathrm{MeOH}$ $(20 \mathrm{ml})$ ] and the resulting solution was stirred at $20^{\circ} \mathrm{C}$ for $30 \mathrm{~min}$, after which time the solvent was removed and the residue taken up in DCM $(30 \mathrm{ml})$ and washed with water and dried. The residue obtained from the work-up was chromatographed using EtOAc:hexane (3:7) as eluent to give the product 11a $(14 \mathrm{mg} ; 81 \%)$ as white cubes, m.p. $179-180{ }^{\circ} \mathrm{C}$; IR $v_{\max }\left(\mathrm{CH}_{2} \mathrm{Cl}_{2}\right): 3250$, 2845, 1640, 1530, $1275 \mathrm{~cm}^{-1} .{ }^{1} \mathrm{H}$ NMR (500 MHz, $\mathrm{CDCl}_{3}$ ): $\delta=2.12,2.21$ and 2.43 (each $3 \mathrm{H}, \mathrm{s}$, $3 \times$ Aryl- $\left.\mathrm{CH}_{3}\right), 3.96\left(\mathrm{~s}, 3 \mathrm{H}, \mathrm{OCH}_{3}\right), 5.05\left(\mathrm{~s}, 2 \mathrm{H}, \operatorname{ArylCH}_{2} \mathrm{OCH}_{2} \mathrm{Ph}\right), 5.08(\mathrm{~s}, 2 \mathrm{H}$, $\left.\operatorname{ArylCH}_{2} \mathrm{OCH}_{2} \mathrm{Ph}\right), 6.01$ (s, $\left.1 \mathrm{H}, 6^{\prime}-\mathrm{H}\right), 6.51$ (s, $\left.1 \mathrm{H}, 4^{\prime}-\mathrm{H}\right), 7.37$ (m, $\left.5 \mathrm{H}, \mathrm{PhCH}_{2} \mathrm{OCH}_{2}\right), 8.40$ (s, $1 \mathrm{H}, \mathrm{PhOH}), 11.87\left(\mathrm{~s}, 1 \mathrm{H}, 3^{\prime}-\mathrm{OH}\right) .{ }^{13} \mathrm{C} \mathrm{NMR}\left(125 \mathrm{MHz}, \mathrm{CDCl}_{3}\right): \delta=8.2,15.6$ and 22.1 (3×Aryl- $\left.\mathrm{CH}_{3}\right), 51.8\left(\mathrm{OCH}_{3}\right), 54.6$ (phenol- $\left.\mathrm{CH}_{2} \mathrm{OCH}_{2} \mathrm{Ph}\right), 70.9\left(\mathrm{PhCH}_{2} \mathrm{OCH}_{2}-\right.$ phenol), 103.7 , $108.0(\mathrm{CH}), 108.1(\mathrm{CH}), 111.0,114.6,127.5(2 \times \mathrm{CH}), 128.2(\mathrm{CH}), 128.7(2 \times \mathrm{CH}), 131.3,134.5$, 136.8, 140.4, 153.5, 157.0, 157.2, 160.5, 172.6 (C=O). HREIMS: $m / z 438.1675$ (calcd. 438.1679 for $\mathrm{C}_{25} \mathrm{H}_{26} \mathrm{O}_{7}$ ).

\section{Synthesis of compounds 12a-d:}

A solution of phomosine A triacetate $(\mathbf{2 a})(100 \mathrm{mg} ; 0.21 \mathrm{mmol})$ in ethanol $(5 \mathrm{ml})$ was treated at $20^{\circ} \mathrm{C}$ with $\mathrm{NaBH}_{4}(16 \mathrm{mg} ; 0.42 \mathrm{mmol})$. After $20 \mathrm{~min}$ the solution became clear. Stirring was continued for a further $30 \mathrm{~min}$ and the solvent was removed to produce a crystalline solid, which was taken up in DCM. The DCM solution was washed with $0.1 \mathrm{M}$ aqueous hydrochloric acid followed by water. After drying $\left(\mathrm{Na}_{2} \mathrm{SO}_{4}\right)$, the solvent was removed under reduced pressure and the residue very carefully chromatographed using EtOAc-hexane (3:7) as eluent to afford the compounds 12a-d in the order of elution:

Methyl 2,4-diacetoxy-5-(3'-acetoxy-2',5'-dimethylphenoxy)-3,6-dimethylbenzoate (12a): (30 mg; $23 \%)$ as white crystals m.p. $151-152{ }^{\circ} \mathrm{C}$. IR $v_{\max }\left(\mathrm{CH}_{2} \mathrm{Cl}_{2}\right): 2840,1640,1535,1270 \mathrm{~cm}^{-1}$. ${ }^{1} \mathrm{H}$ NMR (500 MHz, $\mathrm{CDCl}_{3}$ ): $\delta=1.97,2.02,2.12,2.19(\times 2), 2.30$ and 2.33 (each $3 \mathrm{H}, \mathrm{s}, 4 \times$ Aryl$\mathrm{CH}_{3}$ and $\left.3 \times \mathrm{CH}_{3} \mathrm{CO}\right), 3.91\left(\mathrm{~s}, 3 \mathrm{H}, \mathrm{OCH}_{3}\right), 6.13$ (s, $\left.1 \mathrm{H}, 6^{\prime}-\mathrm{H}\right), 6.53\left(\mathrm{~s}, 1 \mathrm{H}, 4^{\prime}-\mathrm{H}\right) .{ }^{13} \mathrm{C}$ NMR $(125$ $\left.\mathrm{MHz}, \mathrm{CDCl}_{3}\right): \delta=8.9,10.4,13.5,19.9,20.4,20.8,21.1\left(\mathrm{CH}_{3}\right.$ and $\left.\mathrm{CH}_{3} \mathrm{CO}\right), 52.4\left(\mathrm{OCH}_{3}\right), 111.7$, 
$115.9(\mathrm{CH}), 116.5(\mathrm{CH}), 124.6,125.6,129.5,137.0,143.3,143.9,144.0,150.0,155.9,166.3$, 167.5, 168.4, 169.2. EIMS ( $\mathrm{m} / \mathrm{z}) \%=458.2$ (24), 416.1 (54), 374.2 (100), 300.1 (34), 257.2 (8), 211.2 (6), 167.1 (12), 149.0 (20), 83.1 (28). HREIMS: $\mathrm{m} / \mathrm{z} 458.1571$ (calcd. 458.1577 for $\mathrm{C}_{24} \mathrm{H}_{26} \mathrm{O}_{9}$ ).

Methyl 4-acetoxy-5-(3'-acetoxy-2',5'-dimethylphenoxy)-2-hydroxy-3,6-dimethylbenzoate (12b): $(24 \mathrm{mg} ; 27 \%)$ as white crystals m.p. $157-158{ }^{\circ} \mathrm{C}$. IR $\nu_{\max }\left(\mathrm{CH}_{2} \mathrm{Cl}_{2}\right): 3230,2840,1635$, $1535,1275 \mathrm{~cm}^{-1} .{ }^{1} \mathrm{H}$ NMR (500 MHz, $\left.\mathrm{CDCl}_{3}\right): \delta=1.98,2.04,2.13,2.16,2.20$ and 2.30 (each 3 $\mathrm{H}, \mathrm{s}, 4 \times$ Aryl $-\mathrm{CH}_{3}$ and $\left.2 \times \mathrm{CH}_{3} \mathrm{CO}\right), 3.90\left(\mathrm{~s}, 3 \mathrm{H}, \mathrm{OCH}_{3}\right), 5.82\left(\mathrm{~s}, 1 \mathrm{H}, 6^{\prime}-\mathrm{H}\right) 6.30\left(\mathrm{~s}, 1^{\prime} \mathrm{H}, 4^{\prime}-\mathrm{H}\right)$, 11.48 (s, $1 \mathrm{H}, 2-\mathrm{OH}) .{ }^{13} \mathrm{C}$ NMR $\left(125 \mathrm{MHz}, \mathrm{CDCl}_{3}\right): \delta=7.9,10.4,13.5,20.0,20.5,21.2\left(\mathrm{CH}_{3}\right.$ and $\left.\mathrm{CH}_{3} \mathrm{CO}\right), 52.4\left(\mathrm{OCH}_{3}\right), 106.6,109.0(\mathrm{CH}), 110.1(\mathrm{CH}), 124.4,125.5,129.6,136.9,143.4$, 143.9, 144.2, 154.5, 156.0, 166.4. 167.6, 168.5. EIMS $m / z(\%)=416.1$ (26), 388.1 (12), 374.2 (58), 300.1 (100), 286.1 (22), 257.1 (8), 215.1 (5), 149 (10), 83 (23). HREIMS: m/z 416.1469 (calcd. 416.1471 for $\mathrm{C}_{22} \mathrm{H}_{24} \mathrm{O}_{8}$ ).

Methyl 2,4-diacetoxy-5-(3'-hydroxy-2',5'-dimethylphenoxy)-3,6-dimethylbenzoate (12c): (26 mg; 30\%) as white needles m.p. $141-142^{\circ} \mathrm{C}$ (from hexane). IR $v_{\max }\left(\mathrm{CH}_{2} \mathrm{Cl}_{2}\right)$ : 3240, 2840, 1635, 1535, $1275 \mathrm{~cm}^{-1} .{ }^{1} \mathrm{H}$ NMR (500 MHz, $\left.\mathrm{CDCl}_{3}\right): \delta=2.05,2.07,2.13,2.16,2.33$ and 2.36 (each $3 \mathrm{H}, \mathrm{s}, 4 \times$ Aryl- $\mathrm{CH}_{3}$ and $\left.2 \times \mathrm{CH}_{3} \mathrm{CO}\right), 3.98\left(\mathrm{~s}, 3 \mathrm{H}, \mathrm{OCH}_{3}\right), 6.02\left(\mathrm{~s}, 1 \mathrm{H}, 6^{\prime}-\mathrm{H}\right), 6.51(\mathrm{~s}, 1 \mathrm{H}$, $\left.\mathrm{H}-4^{\prime}\right), 11.63$ (s, $\left.1 \mathrm{H}, 3^{\prime}-\mathrm{OH}\right) .{ }^{13} \mathrm{C} \mathrm{NMR}\left(125 \mathrm{MHz}, \mathrm{CDCl}_{3}\right): \delta=8.9,9.4,15.1,20.1,20.8,21.2$ $\left(\mathrm{CH}_{3}\right.$ and $\left.\mathrm{CH}_{3} \mathrm{CO}\right), 52.3\left(\mathrm{OCH}_{3}\right), 110.2(\mathrm{CH}), 111.2,115.7,116.1(\mathrm{CH}), 119.0,131.9,136.8$, 137.8, 147.2, 150.0, 156.5, 159.1, 167.6, 169.2, 172.1. EIMS $m / z=(\%) 416.1$ (78), 374.05 (90), 342.04 (100), 300.05 (84), 257.02 (11), 91.03 (16). HREIMS: $m / z 416.1470$ (calcd. 416.1471 for $\left.\mathrm{C}_{22} \mathrm{H}_{24} \mathrm{O}_{8}\right)$.

Methyl 4-acetoxy-5-(3'-hydroxy-2',5'-dimethylphenoxy)-2-hydroxy-3,6-dimethylbenzoate (12d): (10 mg; 13\%) as white crystals m.p. 204-206 ${ }^{\circ} \mathrm{C}$. IR $\nu_{\max }\left(\mathrm{CH}_{2} \mathrm{Cl}_{2}\right): 3330,2840,1640$, 1535, $1270 \mathrm{~cm}^{-1}$. ${ }^{1} \mathrm{H}$ NMR $\left(500 \mathrm{MHz}, \mathrm{CDCl}_{3}\right.$ ): $\delta=2.07,2.08,2.11,2.22$ and 2.33 (each $3 \mathrm{H}, \mathrm{s}$, 4×Aryl- $\mathrm{CH}_{3}$ and $\left.\mathrm{CH}_{3} \mathrm{CO}\right), 3.97\left(\mathrm{~s}, 3 \mathrm{H}, \mathrm{OCH}_{3}\right), 5.72\left(\mathrm{~s}, 1 \mathrm{H}, 6^{\prime}-\mathrm{H}\right), 6.29$ (s, $\left.1 \mathrm{H}, 4^{\prime}-\mathrm{H}\right), 11.47$ (s, $1 \mathrm{H}, 2-\mathrm{OH}), 11.62$ (s, $\left.1 \mathrm{H}, 3^{\prime}-\mathrm{OH}\right) .{ }^{13} \mathrm{C} \mathrm{NMR}\left(125 \mathrm{MHz}, \mathrm{CDCl}_{3}\right): \delta=7.8,9.4,15.1,20.1,21.3$ $\left(\mathrm{CH}_{3}\right.$ and $\left.\mathrm{CH}_{3} \mathrm{CO}\right), 52.3\left(\mathrm{OCH}_{3}\right), 106.1,108.7(\mathrm{CH}), 109.7(\mathrm{CH}), 110.3,118.8,132.0,136.7$, 137.8, 147.4, 154.4, 156.7, 159.0, 167.7, 172.1. EIMS $\mathrm{m} / \mathrm{z}(5)=374.10$ (90), 332.10 (67), 300.09 (100), 285.04 (15), 179, 02 (4), 148.04 (6), 122.06 (9), 91.04 (14). HREIMS: m/z 374.1365 (calcd. 374.1366 for $\mathrm{C}_{20} \mathrm{H}_{22} \mathrm{O}_{7}$ ).

Agar Diffusion Assay for Biological Activity. The substances were dissolved in acetone at a concentration of $2 \mathrm{mg} / \mathrm{mL}$. Twenty-five microliters of the solutions ( $25 \mu \mathrm{g}$ ) was pipetted onto a sterile filter disk (Schleicher \& Schuell, $9 \mathrm{~mm}$ ), which was placed onto an appropriate agar growth medium for the respective test organism and subsequently sprayed with a suspension of the test organism. ${ }^{9}$ The test organisms were the Gram-positive bacterium Bacillus megaterium and the Gram-negative bacterium Escherichia coli (both grown on NB medium), the fungus Microbotryum violaceum and the alga Chlorella fusca (both grown on MPY medium). Reference substances were penicillin, nystatin, actidione, and tetracycline. Commencing at the middle of 
the filter disk, the radius of the zone of inhibition was measured in millimeters. These microorganisms were chosen because (a) they are nonpathogenic and (b) they had in the past proved to be accurate initial test organisms for antibacterial, antifungal, and antialgal/herbicidal activities.

\section{Acknowledgements}

We thank Jan de Vries for excellent technical assistance and BASF SE for financial support.

\section{References}

1. Cole, R. J. Handbook of Toxic Fungal Metabolites (Ed.: R. H. Cox), Academic Press, New York - London 1981.

2. Krohn, K.; Farooq, U.; Hussain, H.; Rheinheimer, J.; Draeger, S.; Schulz, B. Nat. Prod. Commun. 2011, 5, in press.

3. Krohn, K.; Michel, A.; Roemer, E.; Flörke, U.; Aust, H.-J.; Draeger, S.; Schulz, B.; Wray, V. Nat. Prod. Lett. 1995, 9, 309.

4. Dai, J.; Krohn, K.; Flörke, U.; Gehle, D.; Aust, H.-J.; Draeger, S.; Schulz, B.; Rheinheimer, K. Eur. J. Org. Chem. 2005, 5100.

5. Claridge, T. D. W. High-Resolution NMR Techniques in Organic Chemistry, Pergamon, Oxford 1999.

6. Schorn, C. NMR Spectroscopy: Data Acquisition, Wiley-VCH, Weinheim 2001.

7. Qin, S.; Krohn, K.; Flörke, U.; Schulz, B.; Draeger, S.; Pescitelli, G.; Salvadori, P.; Antus, S.; Kurtán, T. Eur. J. Org. Chem. 2009, 3279.

8. Krohn, K.; Ahmed, I.; John, M.; Letzel, M.; Kuck, D. Eur. J. Org. Chem. 2010, 2544.

9. Höller, U.; Wright, A. D.; Matthée, G. F.; König, G. M.; Draeger, S.; Aust, H.-J.; Schulz, B. Mycolog. Res. 2000, 104, 1354. 